\title{
MOLECULAR ENGINEERING OF CHROMOPHORES TO ENABLE TRIPLET-TRIPLET ANNIHILATION UPCONVERSION
}

\author{
Supporting Information
}

Kealan J. Fallon, Emily M. Churchill, Samuel N. Sanders, James Shee, John L. Weber, Rinat Meir, Steffen Jockusch, David R. Reichman, Matthew Y. Sfeir, ${ }^{*}$ Daniel N. Congreve* \& Luis M. Campos*

2 Optical Properties 14

3 Photon Upconversion 19

4 Transient Absorbance Spectroscopy 24

5 Computational Calculations 26

6 References $\quad 40$ 


\subsection{METHODS}

Unless otherwise stated, consider the following for all reactions: commercial precursors were used as received without further purification; reactions were stirred; reactions were performed under an inert environment (Ar); anhydrous reactions were carried out in oven-dried glassware, cooled under vacuum, and backfilled with Ar.

Anhydrous solvent was obtained from a Schlenk manifold with purification columns packed with activated alumina and supported copper catalyst (Glass Contour, Irvine, CA) or purchased from commercial suppliers. Thin-layer chromatography (TLC) was carried out on Sigma Aldrich Supelco TLC plates and $\mathrm{R}_{\mathrm{F}}$ values are reported for the solvent system used for column chromatography. In cases of gradient chromatography, the $\mathrm{R}_{\mathrm{F}}$ value for the initial eluent mixture is reported. Components on TLC plates were visualised using combinations of UV light and potassium permanganate stain. Column chromatography was carried out using silica.

${ }^{1} \mathrm{H}$ NMR spectra were recorded at either $500 \mathrm{MHz}$ on a Bruker Ascend 500 spectrometer or $400 \mathrm{MHz}$ on a Bruker 400 spectrometer in the stated solvent using residual protic solvent $\mathrm{CDCl}_{3}(\delta=7.26, \mathrm{~s}), \mathrm{d}_{6}$-DMSO $(\delta=2.56, \mathrm{qn}), \mathrm{d}_{6}$-acetone $(\delta=2.05, \mathrm{~s})$ or $\mathrm{D}_{2} \mathrm{O}(\delta=4.79, \mathrm{~s})$ as the internal standard. Chemical shifts $(\delta)$ are quoted in ppm using the following abbreviations: $\mathrm{s}$, singlet; $\mathrm{d}$, doublet; $\mathrm{t}$, triplet; q, quartet; qn, quintet; $\mathrm{m}$, multiplet; br, broad; or a combination of these. Coupling constants $(J)$ are reported in hertz $(\mathrm{Hz}) .{ }^{13} \mathrm{C}$ NMR spectra were recorded at $125 \mathrm{MHz}$ on a Bruker Avance III 500 spectrometer in the stated solvent using the central reference of $\mathrm{CHCl}_{3}(\delta=77.0, \mathrm{t})$ or DMSO $(\delta=39.52$, septet $)$ as the internal standard. Chemical shifts are reported to the nearest $0.1 \mathrm{ppm}$.

Mass spectrometry was performed on a XEVO G2-XS Waters spectrometer equipped with a QTOF detector with multiple inlet and ionization capabilities including electrospray ionization (ESI), atmospheric pressure chemical ionization (APCI), and atmospheric solids analysis probe (ASAP).

Acenequinones (denoted with + ) were not characterised due to their highly insoluble nature, as is common in acene literature.

PdPc (Frontier Scientific), PdTPTBP (Santa Cruz Biotechnology) and Rubrene (Aldrich) were purchased and used as supplied without further purification.

\subsection{SYNTHESIS \& CHARACTERISATION}

\subsubsection{General Procedures}

\section{General Procedure I}

Microwave-Assisted Cyanation of Polycyclic Aromatic Hydrocarbons (PAH)

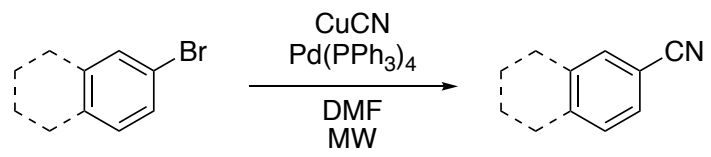

In a dry, degassed microwave vial was added a brominated PAH (300 mg), copper(I) cyanide (3 equiv. per $\mathrm{Br})$ and $\mathrm{Pd}\left(\mathrm{PPh}_{3}\right)_{4}(20 \mathrm{~mol} \%$ per $\mathrm{Br})$ followed by anhydrous degassed $\mathrm{DMF}(15 \mathrm{~mL})$. The suspension was heated at $140^{\circ} \mathrm{C}$ in a microwave reactor for $30 \mathrm{~min}$ per $\mathrm{Br}$. The cooled reaction was poured into water $(15$ $\mathrm{mL}$ ) and the suspension was filtered over an inch of celite. The filtrate was discarded, and the filter cake was 
washed with DCM until washings ran colourless. The filter cake containing excess cyanide was quenched with bleach. The organic filtrate was separated from residual water and washed with brine $(\times 2)$ and dried over $\mathrm{MgSO}_{4}$. The residue obtained after concentration in vacuo was purified by column chromatography on silica gel eluting with $0 \rightarrow 50 \% \mathrm{CHCl}_{3}$ in hexane.

\section{General Procedure II}

TIPS-Acetylation of Acenequinones<smiles></smiles>

To a solution of TIPS-acetylene (3 equiv. to acenequinone) in anhydrous THF $(0.8 \mathrm{M})$ at $0{ }^{\circ} \mathrm{C}$ was added n-butyl lithium ( 2.8 equiv. to acetylene) over $5 \mathrm{~min}$. The reaction was allowed to come to RT. After $1 \mathrm{~h}$, an acenequinone ( 1 equiv.) was added in one portion and the reaction stirred overnight. The reaction was quenched and diluted with wet THF and poured into a rapidly stirred solution of stannous chloride dihydrate (10 equiv. to quinone) in $10 \%$ hydrochloric acid $(5 \mathrm{w} / \mathrm{t}$ equiv.). After $1 \mathrm{~h}$, the reaction was extracted with DCM $(\times 3)$, the combined extracts washed with water $(\times 3)$, brine and dried over $\mathrm{MgSO}_{4}$. The solution was finally filtered through an inch of celite to remove residual stannous impurities. The residue obtained after concentration in vacuo was purified by column chromatography on silica gel.

\section{General Procedure III}

Formation of acenequinones<smiles>[R][Y17]1ccc2c(c1)C(=O)C=CC2=O</smiles>

A solution of a tetrabromide, a naphthoquinone ( 1 equiv.) and potassium iodide ( 4 equiv.) in anhydrous DMF $(0.1 \mathrm{M})$ was heated at $110^{\circ} \mathrm{C}$ overnight, forming a precipitate. The reaction was cooled, diluted with methanol (equal volume to DMF) and filtered. The filter cake was washed sequentially with water, methanol, DCM and air-dried.

\subsubsection{Tetracenes}

\section{6,11-Bis ((triisopropylsilyl)ethynyl)tetracene-2-carbonitrile (1)} viz. Tc-CN1

Using general procedure I and ((8-bromotetracene-5,12-diyl)bis(ethyne-2,1diyl))bis(triisopropylsilane $)^{[1]} \quad(300 \mathrm{mg}, \quad 0.45 \mathrm{mmol}), \quad \mathrm{CuCN} \quad(121 \mathrm{mg}$, $1.35 \mathrm{mmol})$ and $\mathrm{Pd}\left(\mathrm{PPh}_{3}\right)_{4}(104 \mathrm{mg}, 20 \mathrm{~mol} \%)$ in $\mathrm{DMF}(15 \mathrm{~mL})$ gave the title compound as a red solid $(226 \mathrm{mg}, 82 \%)$.

${ }^{1} \mathrm{H}$ NMR $\left(500 \mathrm{MHz}, \mathrm{CDCl}_{3}\right) \delta 9.38(\mathrm{~s}, 1 \mathrm{H}), 9.34(\mathrm{~s}, 1 \mathrm{H}), 8.67-8.60(\mathrm{~m}, 2 \mathrm{H})$, $8.44(\mathrm{~s}, 1 \mathrm{H}), 8.07(\mathrm{~d}, J=8.9 \mathrm{~Hz}, 1 \mathrm{H}), 7.65-7.58(\mathrm{~m}, 2 \mathrm{H}), 7.50(\mathrm{dd}, J=8.9,1.5$ $\mathrm{Hz}, 1 \mathrm{H}), 1.37-1.29(\mathrm{~m}, 42 \mathrm{H}){ }^{13} \mathrm{C}$ NMR $(126 \mathrm{MHz}, \mathrm{CDCl} 3) \delta 136.7,133.6$,

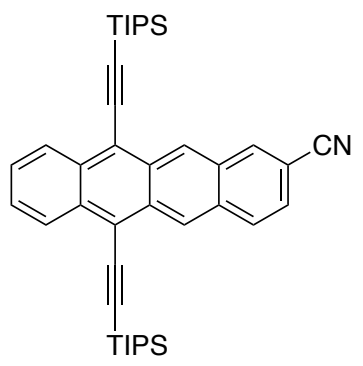


133.3, 131.6, 131.5, 130.8, 130.4, 130.1, 128.6, 127.8, 127.6, 127.3, 124.7, 119.6, 119.5, 119.1, 109.6, 107.4, 107.0, 103.4, 103.3, 19.1, 11.7 HRMS Found (ASAP+): $[\mathrm{M}+\mathrm{H}]^{+}$614.3627, $\mathrm{C}_{41} \mathrm{H}_{52} \mathrm{NSi}_{2}$ requires 614.3638
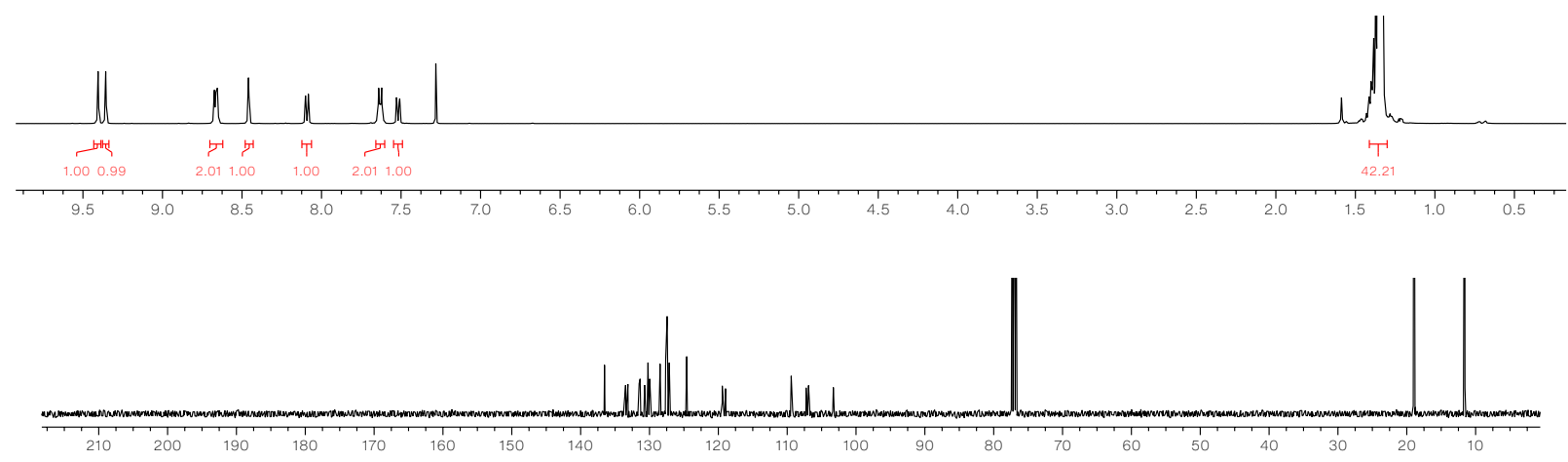

((8,9-Dibromotetracene-5,12-diyl)bis(ethyne-2,1diyl))bis(triisopropylsilane) (2)

Using general procedure II and 8,9-dibromotetracene-5,12-dione ${ }^{[2]}(1 \mathrm{~g}, 2.4$ $\mathrm{mmol}$ ) gave the title compound as a purple solid $(1.1 \mathrm{~g}, 61 \%)$.

${ }^{1} \mathrm{H}$ NMR $\left(500 \mathrm{MHz}, \mathrm{CDCl}_{3}\right) \delta 9.18(\mathrm{~s}, 2 \mathrm{H}), 8.61(\mathrm{dd}, J=6.8,3.2 \mathrm{~Hz}, 2 \mathrm{H}), 8.31$ $(\mathrm{s}, 2 \mathrm{H}), 7.57$ (dd, $J=6.8,3.2 \mathrm{~Hz}, 2 \mathrm{H}), 1.34-1.28(\mathrm{~m}, 42 \mathrm{H}){ }^{13} \mathbf{C}$ NMR (125

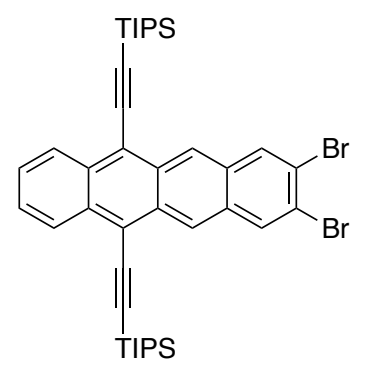
$\left.\mathrm{MHz}, \mathrm{CDCl}_{3}\right) \delta 133.3,132.8,131.2,130.8,127.6,127.4,125.9,122.4,119.0$, 106.8, 103.5, 19.1, 11.7 HRMS Found (ASAP+): $[\mathrm{M}]^{+}$744.1811, $\mathrm{C}_{40} \mathrm{H}_{50} \mathrm{Br}_{2} \mathrm{Si}_{2}$ requires 744.1818

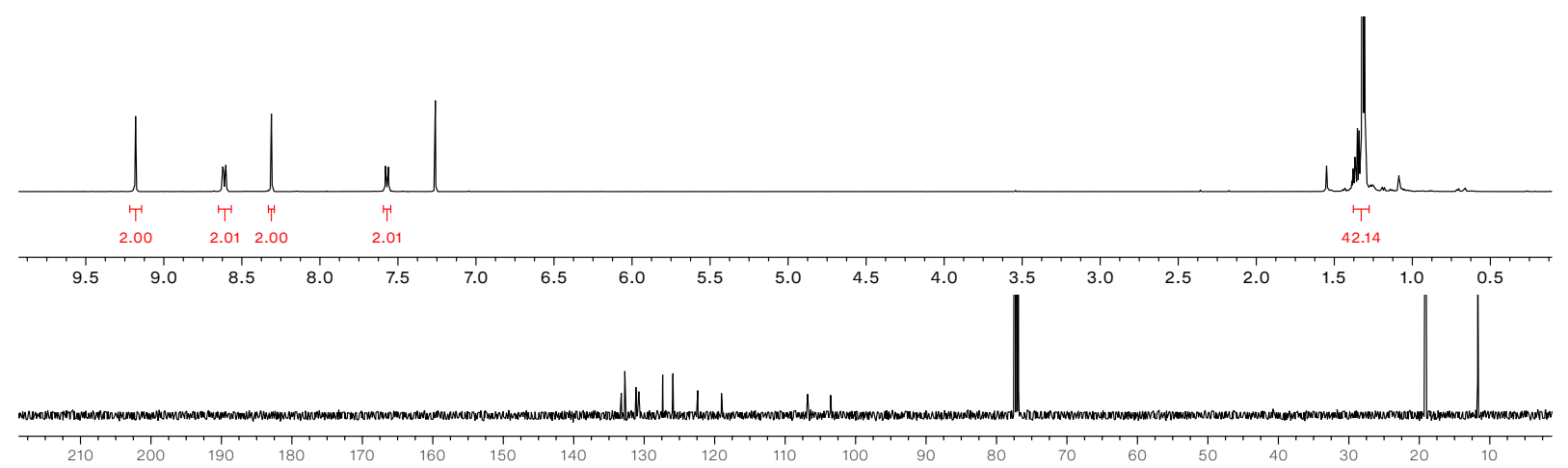

((8,9-Dibromotetracene-5,12-diyl)bis(ethyne-2,1-

diyl))bis(triisopropylsilane) (3)

viz. Tc-CN2ZT

Using general procedure I and dibromotetracene $2(300 \mathrm{mg}, 0.4 \mathrm{mmol}), \mathrm{CuCN}$ (216 mg, $2.4 \mathrm{mmol}), \mathrm{Pd}\left(\mathrm{PPh}_{3}\right)_{4}(185 \mathrm{mg}, 40 \mathrm{~mol} \%)$ in $\mathrm{DMF}(15 \mathrm{~mL})$ gave the title compound as a red solid (171 mg, 67\%).

${ }^{1} \mathrm{H}$ NMR $\left(500 \mathrm{MHz}, \mathrm{CDCl}_{3}\right) \delta 9.42(\mathrm{~s}, 2 \mathrm{H}), 8.67(\mathrm{dd}, J=6.7,3.2 \mathrm{~Hz}, 2 \mathrm{H}), 8.50$ (s, 2H), $7.68(\mathrm{dd}, J=6.7,3.2 \mathrm{~Hz}, 2 \mathrm{H}), 1.42-1.29(\mathrm{~m}, 42 \mathrm{H}){ }^{13} \mathrm{C}$ NMR $(125$ $\left.\mathrm{MHz}, \mathrm{CDCl}_{3}\right) \delta 138.7,134.1,131.9,129.5,129.2,128.5,127.7,120.0,116.3$,

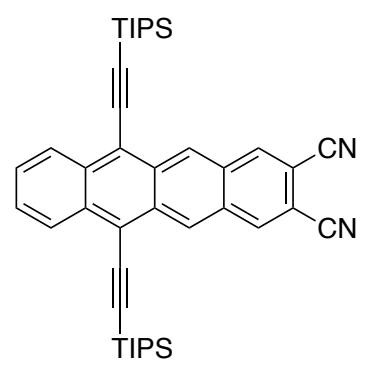


108.5, 102.7, 19.1, 11.7 HRMS Found (ESI+): $[\mathrm{M}+\mathrm{Na}]^{+}$661.3409, $\mathrm{C}_{42} \mathrm{H}_{50} \mathrm{~N}_{2} \mathrm{NaSi}_{2}$ requires 661.3410
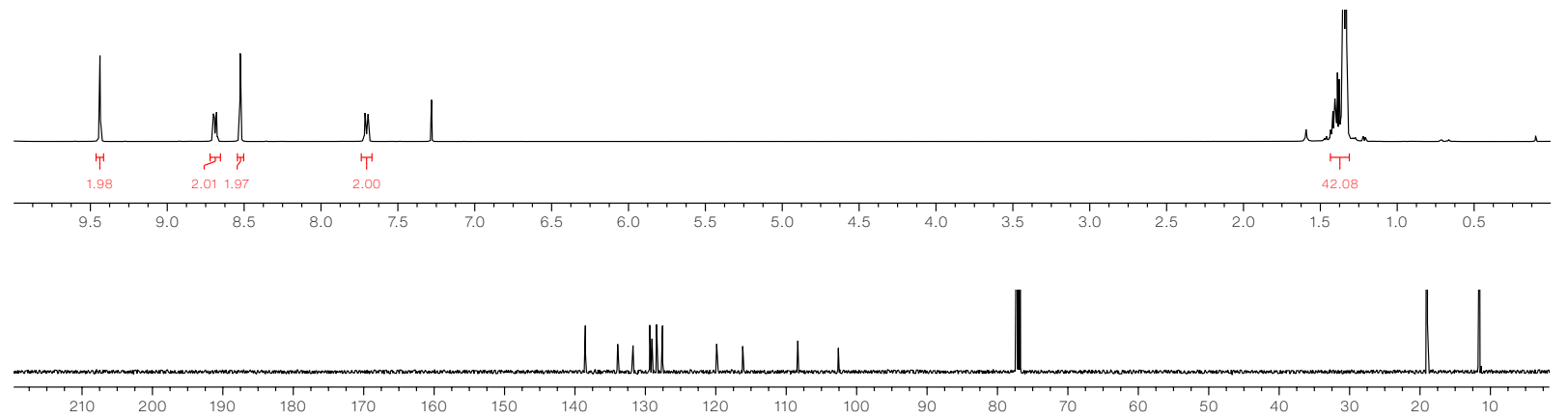

5,12-Bis ((triisopropylsilyl)ethynyl)tetracene-2,(8/9)-dicarbonitrile (4) viz. Tc-CN2E

Using general procedure I and a 1:1 mixture of ((2,8/9-dibromotetracene-5,12diyl)bis(ethyne-2,1-diyl))bis(triisopropylsilane) $\mathrm{s}^{[3]} \quad$ (300 mg, $0.4 \mathrm{mmol}$ ), $\mathrm{CuCN}(215 \mathrm{mg}, 2.4 \mathrm{mmol})$ and $\mathrm{Pd}\left(\mathrm{PPh}_{3}\right)_{4}(185 \mathrm{mg}, 40 \mathrm{~mol} \%)$ in DMF (20 $\mathrm{mL}$ ) gave a 1:1 mixture of the title compounds as a red solid (192 mg, 75\%).

${ }^{1} \mathrm{H}$ NMR $\left(500 \mathrm{MHz}, \mathrm{CDCl}_{3}\right) \delta 9.41(\mathrm{~d}, J=3.0 \mathrm{~Hz}, 2 \mathrm{H}), 9.37(\mathrm{~d}, J=3.2 \mathrm{~Hz}, 2 \mathrm{H})$, $9.08(\mathrm{~s}, 2 \mathrm{H}), 8.72(\mathrm{dd}, J=9.0,0.9 \mathrm{~Hz}, 2 \mathrm{H}), 8.48(\mathrm{~d}, J=4.0,2 \mathrm{H}), 8.12(\mathrm{dd}, J=$ 9.0, 4.0, 2H), $7.66(\mathrm{dt}, J=9.0,2.2 \mathrm{~Hz}, 2 \mathrm{H}), 7.58(\mathrm{dt}, J=8.8,1.8 \mathrm{~Hz}, 2 \mathrm{H}), 1.45-$ $1.30(\mathrm{~m}, 84 \mathrm{H}){ }^{13} \mathbf{C} \mathbf{N M R}\left(125 \mathrm{MHz}, \mathrm{CDCl}_{3}\right) \delta 136.4,135.1,135.0,132.9,132.6$, $132.3,131.9,131.7,131.5,131.2,130.9,130.9,130.5,130.3,129.1,128.7,127.8$, $127.5,126.3,126.2,125.5,125.4,121.3,120.8,120.2,119.7,119.0,118.9,111.1$, 110.9, 110.4, 110.3, 110.2, 109.8, 109.0, 108.7, 102.3, 102.2, 102.0, 101.9, 18.9, 11.5 HRMS Found (ASAP+): $[\mathrm{M}+\mathrm{H}]^{+}$639.3590, $\mathrm{C}_{42} \mathrm{H}_{51} \mathrm{~N}_{2} \mathrm{NaSi}_{2}$ requires 661.3591

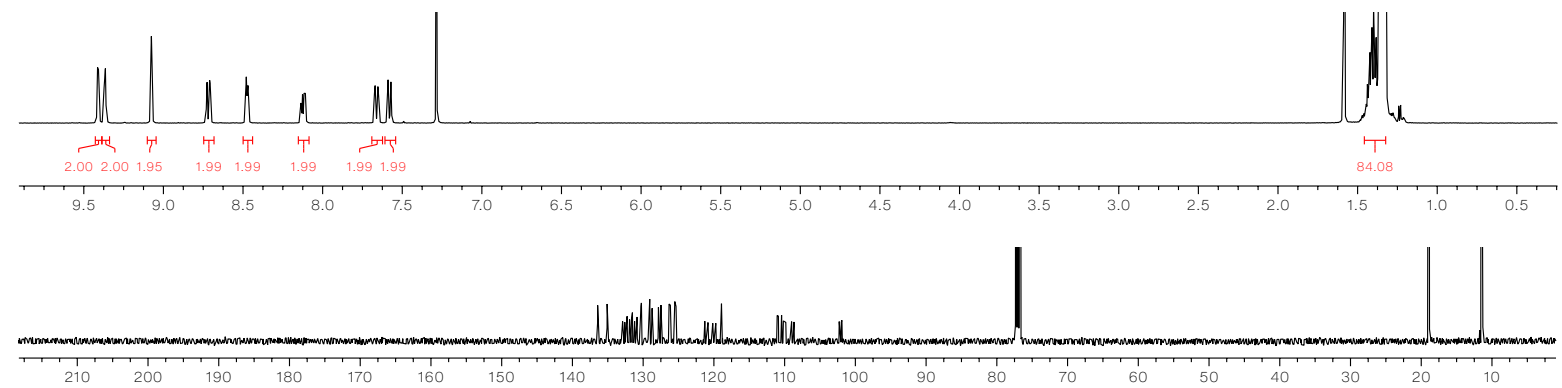

((2,8,9-Tribromotetracene-5,12-diyl)bis(ethyne-2,1diyl))bis(triisopropylsilane) (5)

Using general procedure III, 6-bromonaphthalene-1,4-dione (1.5 g, $6.3 \mathrm{mmol}$ ), 1,2-dibromo-4,5-bis(dibromomethyl)benzene (3.7 g, 6.3), potassium iodide

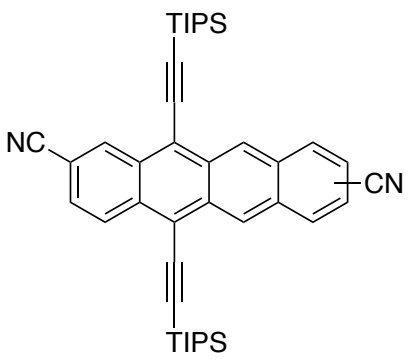
$(4.2 \mathrm{~g}, 25.2 \mathrm{mmol})$ in DMF $(75 \mathrm{~mL})$ gave the title compound as a yellow solid $(1.4 \mathrm{~g}, 45 \%)$. 
${ }^{+}$This compound was not characterised

((2,8,9-Tribromotetracene-5,12-diyl)bis(ethyne-2,1-

diyl))bis(triisopropylsilane) (6)

Using general procedure II and compound $\mathbf{5}(1.4 \mathrm{~g}, 2.8 \mathrm{mmol})$ gave the title compound as a purple solid $(2.2 \mathrm{~g}, 98 \%)$.

${ }^{1} \mathrm{H}$ NMR $\left(500 \mathrm{MHz}, \mathrm{CDCl}_{3}\right) \delta 9.18(\mathrm{~s}, 2 \mathrm{H}), 8.61(\mathrm{dd}, J=6.8,3.2 \mathrm{~Hz}, 2 \mathrm{H}), 8.31$ (s, 2H), 7.57 (dd, $J=6.8,3.2 \mathrm{~Hz}, 2 \mathrm{H}), 1.34-1.28(\mathrm{~m}, 42 \mathrm{H}){ }^{13} \mathbf{C}$ NMR $(125$<smiles></smiles>
$\left.\mathrm{MHz}, \mathrm{CDCl}_{3}\right) \delta 133.3,132.8,131.2,130.8,127.6,127.4,125.9,122.4,119.0$, 106.8, 103.5, 19.1, 11.7 HRMS Found (ASAP+): $[\mathrm{M}]^{+} 822.0919, \mathrm{C}_{40} \mathrm{H}_{49} \mathrm{Br}_{3} \mathrm{Si}_{2}$ requires 822.0923

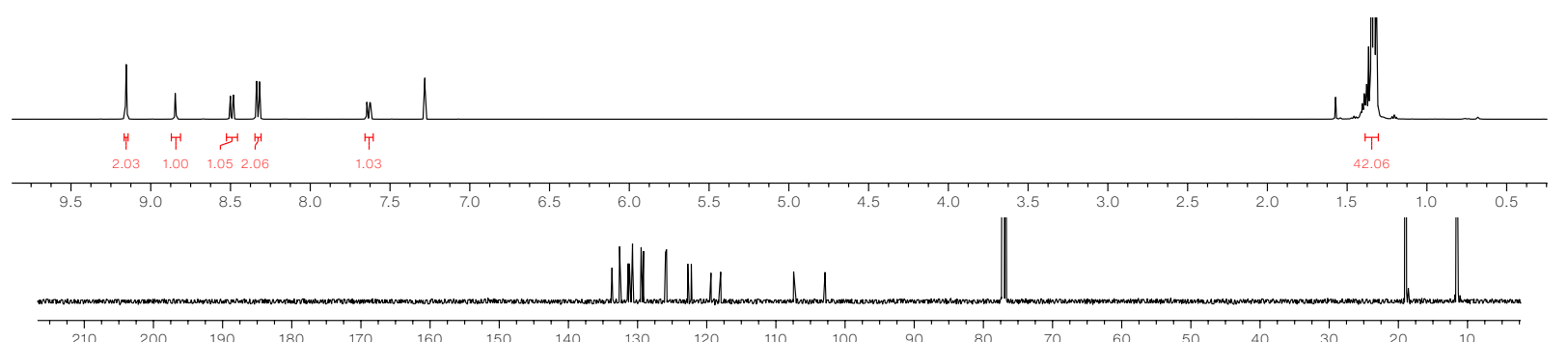

\section{6,11-Bis $(($ triisopropylsilyl)ethynyl)tetracene-2,3,8-tricarbonitrile (7)}

Using general procedure I and tribromotetracene $6(300 \mathrm{mg}, 0.36 \mathrm{mmol})$, $\mathrm{CuCN}(300 \mathrm{mg}, 3.3 \mathrm{mmol})$ and $\mathrm{Pd}\left(\mathrm{PPh}_{3}\right)_{4}(245 \mathrm{mg}, 60 \mathrm{~mol} \%)$ in DMF (20 $\mathrm{mL}$ ) gave the title compound as a red solid (165 mg, 69\%).

${ }^{1} \mathrm{H}$ NMR (500 MHz, $\left.\mathrm{CDCl}_{3}\right) \delta 9.45(\mathrm{~s}, 2 \mathrm{H}), 9.09(\mathrm{~s}, 1 \mathrm{H}), 8.75(\mathrm{~d}, J=9.0 \mathrm{~Hz}$, $1 \mathrm{H}), 8.56(\mathrm{~d}, J=5.3 \mathrm{~Hz}, 2 \mathrm{H}), 7.74(\mathrm{dd}, J=9.0,1.5 \mathrm{~Hz}, 1 \mathrm{H}), 1.52-1.24(\mathrm{~m}$,

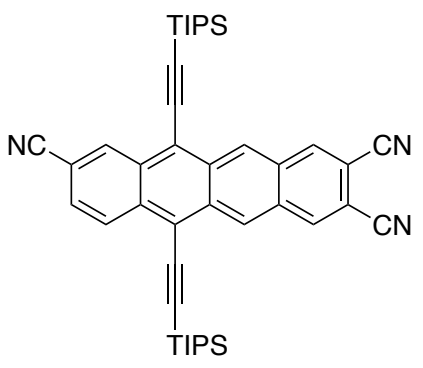
$42 \mathrm{H}){ }^{13} \mathbf{C}$ NMR $\left(125 \mathrm{MHz}, \mathrm{CDCl}_{3}\right) \delta 138.4,138.4,135.0,133.4,132.6,132.0$, 130.0, 129.7, 129.6, 129.2, 127.1, 121.6, 120.6, 118.6, 115.9, 111.8, 111.3, 110.2, $109.4,109.3,101.7,101.4,18.9,11.5$. HRMS Found $(\mathrm{APCI}+):[\mathrm{M}+\mathrm{H}]^{+}$ 664.3549, $\mathrm{C}_{43} \mathrm{H}_{50} \mathrm{~N}_{3} \mathrm{Si}_{2}$ requires 664.3543
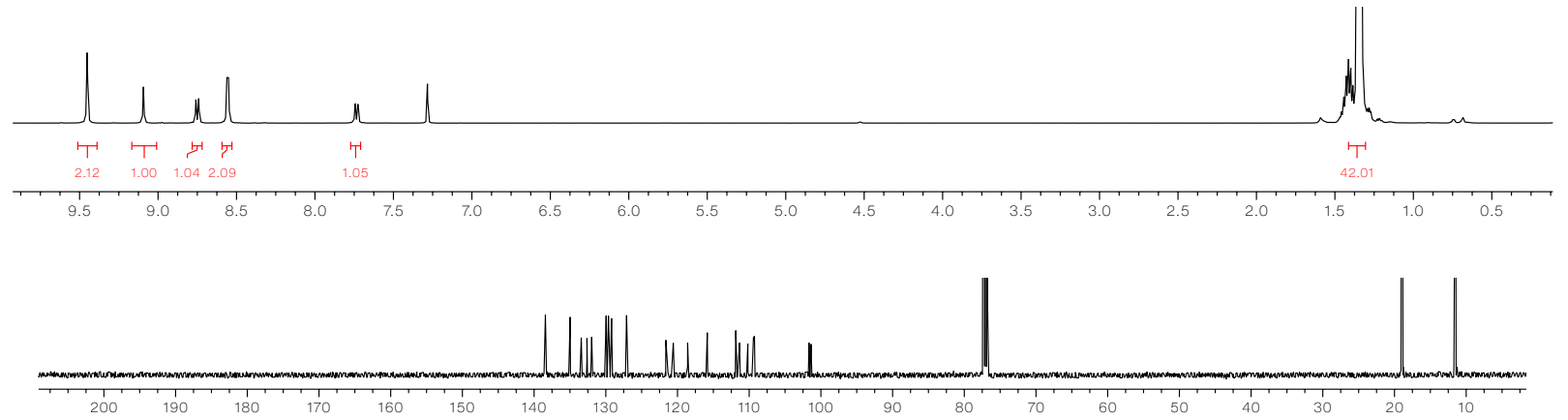
2,3,8,9-Tetrabromotetracene-5,12-dione (8)

Using general procedure III, 6,7-dibromonaphthalene-1,4-dione (2 g, 6.3 $\mathrm{mmol}$ ), 1,2-dibromo-4,5-bis(dibromomethyl)benzene ( $3.7 \mathrm{~g}, 6.3$ ), potassium iodide $(4.2 \mathrm{~g}, 25.2 \mathrm{mmol})$ in DMF $(75 \mathrm{~mL})$ gave the title compound as a yellow solid $(1.8 \mathrm{~g}, 51 \%)$.

${ }^{+}$This compound was not characterised

((2,3,8,9-Tetrabromotetracene-5,12-diyl)bis(ethyne-2,1-

diyl))bis(triisopropylsilane) (9)

Using general procedure II and compound $8(1.3 \mathrm{~g}, 2.3 \mathrm{mmol})$ gave the title compound as a purple solid ( $1.68 \mathrm{~g}, 82 \%)$.

${ }^{1} \mathrm{H}$ NMR $\left(500 \mathrm{MHz}, \mathrm{CDCl}_{3}\right) \delta 9.13(\mathrm{~s}, 2 \mathrm{H}), 8.97$ (s, 2H), $8.33(\mathrm{~s}, 2 \mathrm{H}), 1.43-$ $1.29(\mathrm{~m}, 42 \mathrm{H}){ }^{13} \mathbf{C ~ N M R}\left(125 \mathrm{MHz}, \mathrm{CDCl}_{3}\right) \delta 132.6,132.2,131.9,131.4,130.6$, 126.0, 124.2, 123.0, 118.2, 108.1, 102.5, 18.9, 11.5. HRMS Found (ASAP+): $[\mathrm{M}]^{+}$900.0046, $\mathrm{C}_{40} \mathrm{H}_{48} \mathrm{Br}_{4} \mathrm{Si}_{2}$ requires 900.0028<smiles>O=C1c2cc(Br)c(Br)cc2C(=O)c2cc3cc(Br)c(Br)cc3cc21</smiles>

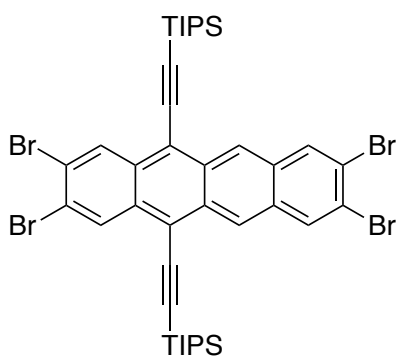

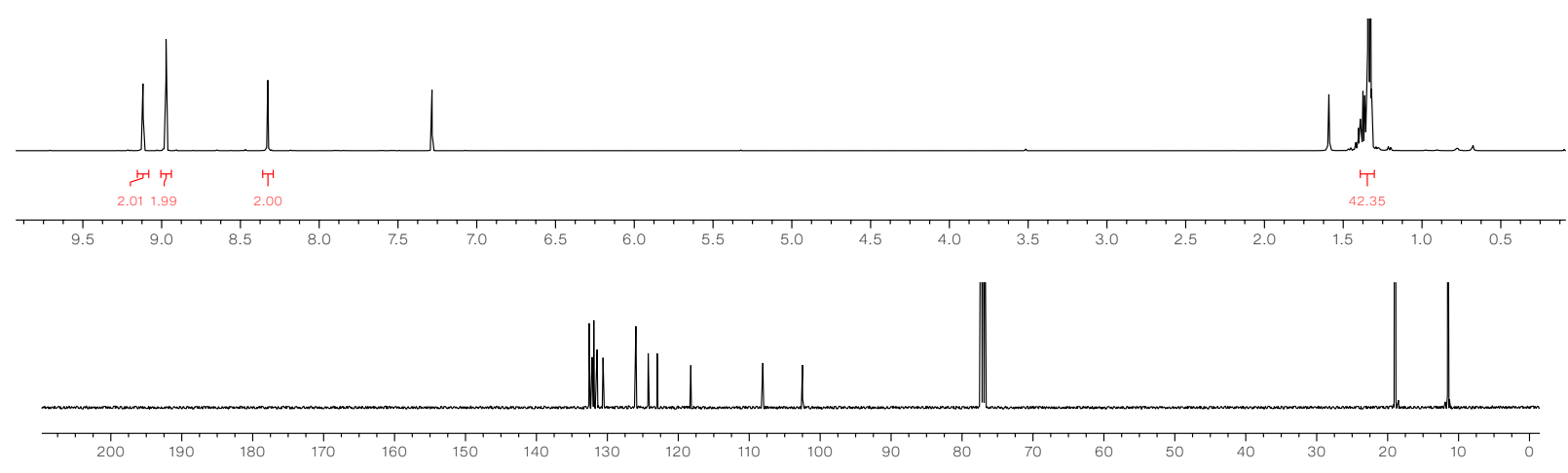

5,12-Bis ((triisopropylsilyl)ethynyl)tetracene-2,3,8,9-tetracarbonitrile (10)

Using general procedure I and tetrabromotetracene $9(200 \mathrm{mg}, 0.22 \mathrm{mmol})$, $\mathrm{CuCN}(236 \mathrm{mg}, 2.6 \mathrm{mmol})$ and $\mathrm{Pd}\left(\mathrm{PPh}_{3}\right)_{4}(204 \mathrm{mg}, 80 \mathrm{~mol} \%)$ in DMF (15 $\mathrm{mL}$ ) gave the title compound as a purple solid ( $84 \mathrm{mg}, 55 \%$ ).

${ }^{1} \mathrm{H}$ NMR ( $\left.500 \mathrm{MHz}, \mathrm{CDCl}_{3}\right) \delta 9.47(\mathrm{~s}, 2 \mathrm{H}), 9.16(\mathrm{~s}, 2 \mathrm{H}), 8.59(\mathrm{~s}, 2 \mathrm{H}), 1.48$ $1.39(\mathrm{~m}, 6 \mathrm{H}), 1.35(\mathrm{~d}, J=7.1 \mathrm{~Hz}, 36 \mathrm{H}){ }^{13} \mathbf{C} \mathbf{N M R}\left(125 \mathrm{MHz}, \mathrm{CDCl}_{3}\right) \delta 138.3$,

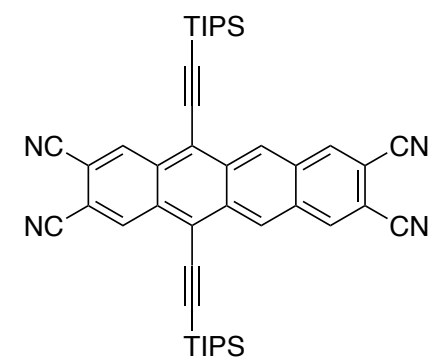
137.0, 132.7, 131.4, 130.6, 130.2, 122.4, 115.5, 113.2, 110.9, 110.3, 100.5, 18.9, 11.5 HRMS Found (ESI+): $[\mathrm{M}+\mathrm{Na}]^{+} 711.3286, \mathrm{C}_{40} \mathrm{H}_{48} \mathrm{Br}_{4} \mathrm{Si}_{2}$ requires 711.3315

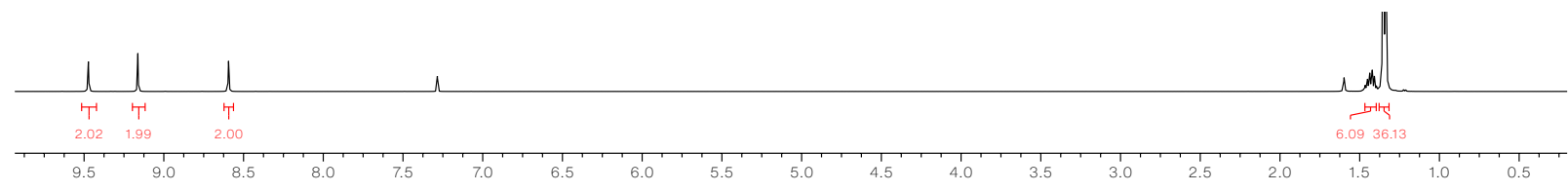




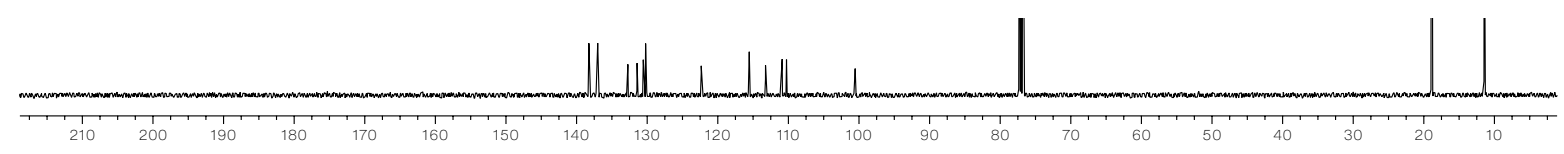

((8,9-Dibromotetracene-5,12-diyl)bis(ethyne-2,1diyl))bis(triisopropylsilane) (11)

Using general procedure II and 8,9-dibromotetracene-5,12-dione ${ }^{[2]}(1 \mathrm{~g}, 2.4$ $\mathrm{mmol}$ ) gave the title compound as a purple solid $(1.1 \mathrm{~g}, 61 \%)$.

${ }^{1} \mathrm{H}$ NMR $\left(500 \mathrm{MHz}, \mathrm{CDCl}_{3}\right) \delta 9.26(\mathrm{~s}, 2 \mathrm{H}), 8.99(\mathrm{~s}, 2 \mathrm{H}), 8.04(\mathrm{dd}, J=6.5,3.2$ $\mathrm{Hz}, 2 \mathrm{H}), 7.52(\mathrm{dd}, J=6.5,3.2 \mathrm{~Hz}, 2 \mathrm{H}), 1.45-1.29(\mathrm{~m}, 42 \mathrm{H}){ }^{13} \mathbf{C}$ NMR $(125$

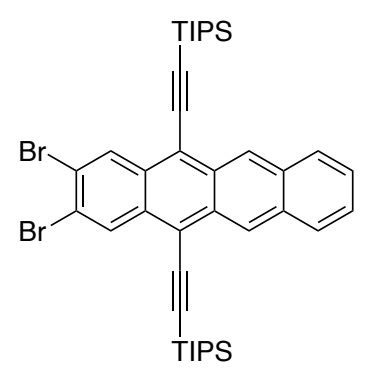
$\left.\mathrm{MHz}, \mathrm{CDCl}_{3}\right) \delta 132.6,131.9,131.7,130.3,128.6,126.6,126.5,123.5,118.0$, 107.2, 103.0, 18.9, 11.6 HRMS Found (ASAP+): $[\mathrm{M}+\mathrm{H}]^{+}$745.1886, $\mathrm{C}_{40} \mathrm{H}_{51} \mathrm{Br}_{2} \mathrm{Si}_{2}$ requires 745.1896

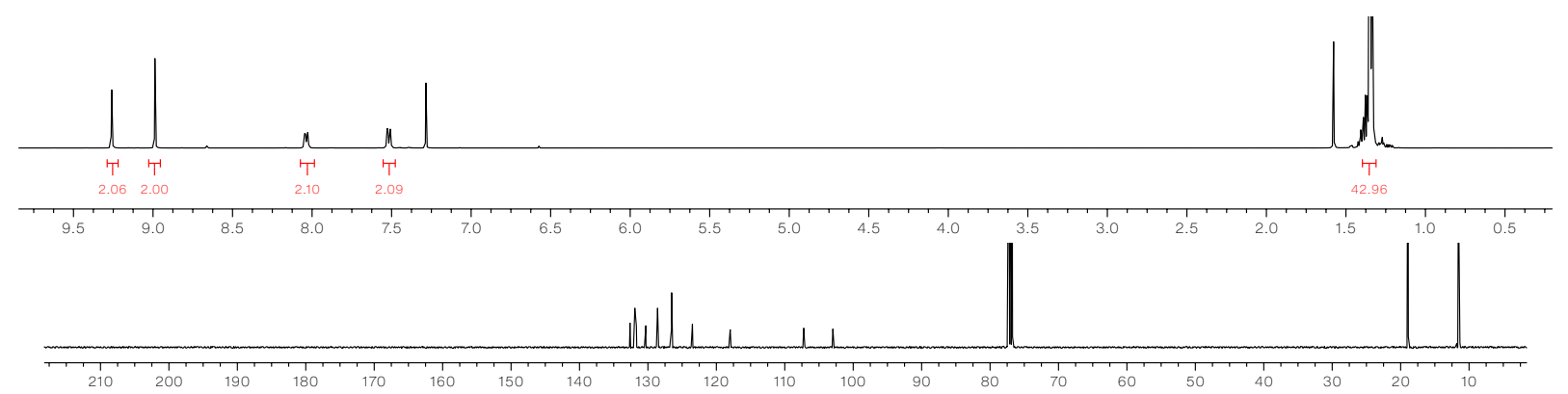

5,12-Bis ((triisopropylsilyl)ethynyl)tetracene-2,3-dicarbonitrile (12)

Using general procedure I and dibromotetracene 11 (275 mg, $0.37 \mathrm{mmol})$, $\mathrm{CuCN}$ (198 mg, $2.2 \mathrm{mmol}), \mathrm{Pd}\left(\mathrm{PPh}_{3}\right)_{4}(171 \mathrm{mg}, 40 \mathrm{~mol} \%)$ in DMF $(15 \mathrm{~mL})$ gave the title compound as a red solid (135 mg, 57\%).

${ }^{1} \mathrm{H}$ NMR $\left(500 \mathrm{MHz}, \mathrm{CDCl}_{3}\right) \delta 9.44(\mathrm{~s}, 2 \mathrm{H}), 8.69(\mathrm{dd}, J=6.7,3.3 \mathrm{~Hz}, 2 \mathrm{H}), 8.53$ $(\mathrm{s}, 2 \mathrm{H}), 7.70(\mathrm{dd}, J=6.7,3.3 \mathrm{~Hz}, 2 \mathrm{H}), 1.43-1.31(\mathrm{~m}, 42 \mathrm{H}){ }^{13} \mathbf{C}$ NMR $(125$<smiles>N#Cc1cc2c(C#C[In])c3cc4ccccc4cc3c(C#C[In])c2cc1C#N</smiles>
$\left.\mathrm{MHz}, \mathrm{CDCl}_{3}\right) \delta 137.2,133.6,131.1,130.0,128.7,127.7,127.5,121.4,116.2$, 110.4, 109.0, 101.6, 18.9, 11.5 HRMS Found (ESI+): $[\mathrm{M}+\mathrm{Na}]^{+}$661.3419, $\mathrm{C}_{42} \mathrm{H}_{50} \mathrm{~N}_{2} \mathrm{NaSi}_{2}$ requires 661.3410
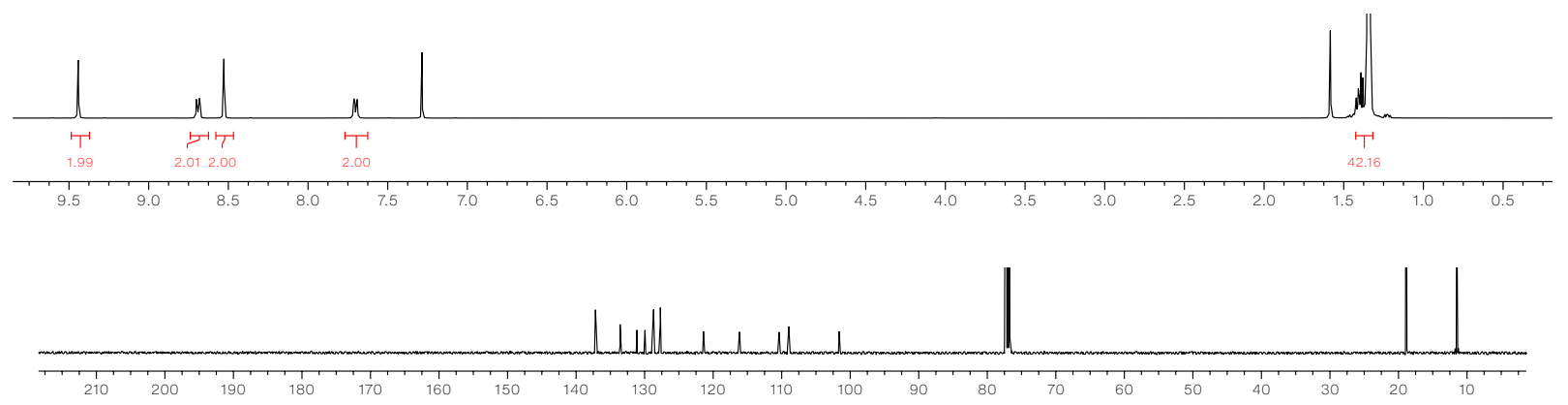


\subsubsection{Perylenes}

\section{2,5,8,11-tetra-tert-butylperylene (13)}

Synthesis of the title compound was adapted from literature procedure. ${ }^{[4]}$ Perylene ( $2 \mathrm{~g}, 7.9 \mathrm{mmol}, 1$ equiv.) and aluminium chloride ( $4 \mathrm{~g}, 30 \mathrm{mmol}, 3.8$ equiv.) were dissolved in tert-butylchloride $(200 \mathrm{~mL})$ and refluxed $55^{\circ} \mathrm{C}$ for $6 \mathrm{~h}$, after which a second addition of tert-butylchloride $(120 \mathrm{~mL})$ was added and the reaction was refluxed for an additional $16 \mathrm{~h}$. A second addition of aluminium chloride ( $4 \mathrm{~g}, 30 \mathrm{mmol}, 3.8$ equiv.) and a third addition of tert-butylchloride (80 $\mathrm{mL}$ ) were added simultaneously and the reaction was left to reflux for $24 \mathrm{~h}$. Once cooled to RT, the crude mixture was washed with brine $(200 \mathrm{~mL})$. The aqueous layer was extracted with DCM $(4 \times 100 \mathrm{~mL})$. All organic layers were collected, dried with $\mathrm{Na}_{2} \mathrm{SO}_{4}$, and concentrated in vacuo to a brown liquid. Crude solid was obtained after baking the residue in a petri dish on a hot plate at $175^{\circ} \mathrm{C}$ for $24 \mathrm{~h}$. This solid residue was purified by column chromatography on silica gel eluting with $10 \%$ chloroform in hexanes, and finally washed with methanol to afford the title compound as a yellow solid (2.65 g, 70\%).

${ }^{1} \mathbf{H}$ NMR $\left(500 \mathrm{MHz}, \mathrm{CDCl}_{3}\right) \delta 8.24(\mathrm{~d}, J=1.7 \mathrm{~Hz}, 4 \mathrm{H}), 7,62(\mathrm{~d}, J=1.6 \mathrm{~Hz}, 4 \mathrm{H})$, $1.50(\mathrm{~s}, 36 \mathrm{H})$ HRMS Found (ASAP+): $[\mathrm{M}+\mathrm{H}]^{+} 477.3514, \mathrm{C}_{36} \mathrm{H}_{45}$ requires 477.3521

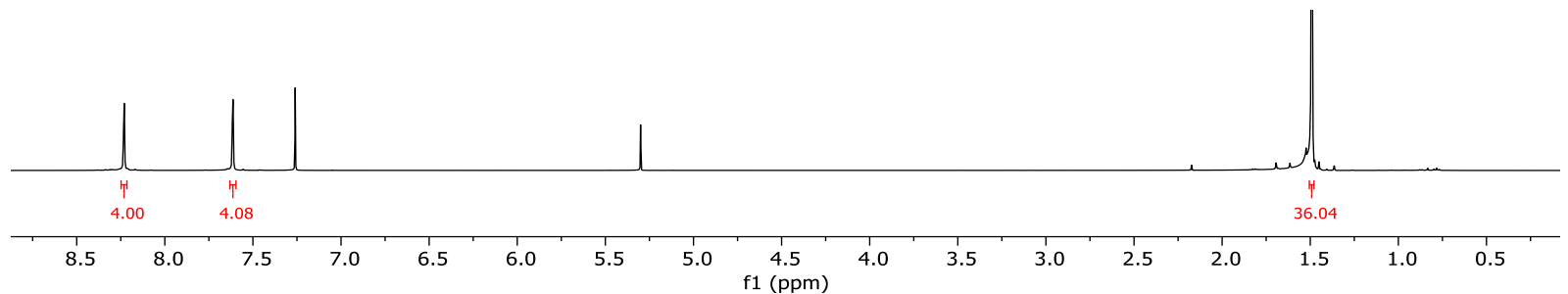

\section{3-Bromo-2,5,8,11-tetra-tert-butylperylene (14)}

A solution of 13 (200 mg, $0.42 \mathrm{mmol}, 1$ equiv.) and $\mathrm{N}$-bromosuccinimide ( 89 $\mathrm{mg}, 0.5 \mathrm{mmol}, 1.2$ equiv. ) in DMF $(12 \mathrm{~mL})$ was stirred at room temperature for $16 \mathrm{~h}$. The mixture was diluted with water $(40 \mathrm{~mL})$ and extracted with DCM $(3 \times 30 \mathrm{~mL})$. The organic layers were collected, washed with water $(5 \times 50 \mathrm{~mL})$, dried with $\mathrm{Na}_{2} \mathrm{SO}_{4}$, and concentrated. Silica column chromatography, with<smiles>CC(C)(C)C1=CC2=c3c(Br)cc(C(C)(C)C)cc3=c3c(Br)cc(C(C)(C)C)cc3=c3c2cc(C(C)(C)C)cc3=C1</smiles>
hexanes as eluent, afforded the title compound as a yellow solid (145 mg, 62\%).

${ }^{1}$ H NMR $\left(500 \mathrm{MHz}, \mathrm{CDCl}_{3}\right) \delta 8.41(\mathrm{~d}, \mathrm{~J}=1.7 \mathrm{~Hz}, 1 \mathrm{H}), 8.36(\mathrm{~s}, 1 \mathrm{H}), 8.30(\mathrm{~d}, \mathrm{~J}$ $=1.7 \mathrm{~Hz}, 1 \mathrm{H}), 8.27(\mathrm{dd}, \mathrm{J}=4.1,1.8 \mathrm{~Hz}, 1 \mathrm{H}), 8.23(\mathrm{~d}, \mathrm{~J}=1.8 \mathrm{~Hz}, 1 \mathrm{H}), 7.65(\mathrm{t}, \mathrm{J}$ $=1.8 \mathrm{~Hz}, 2 \mathrm{H}), 1.74(\mathrm{~s}, 9 \mathrm{H}), 1.55-1.47(\mathrm{~m}, 27 \mathrm{H})$. HRMS Found (ASAP+): $[\mathrm{M}+\mathrm{H}]^{+}$557.2618, $\mathrm{C}_{36} \mathrm{H}_{44} \mathrm{Br}$ requires 557.2612 


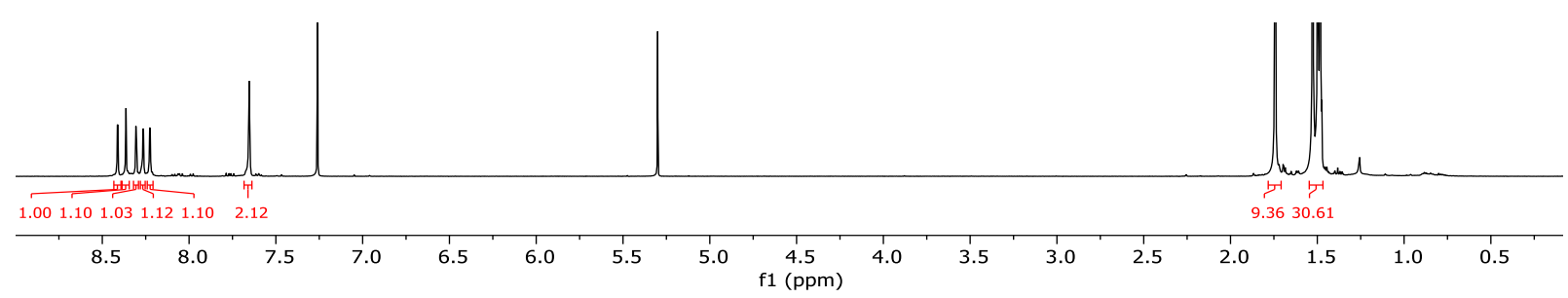

\section{3-Cyano-2,5,8,11-tetra-tert-butylperylene (15)}

Using general procedure I and bromide 14 (100 mg, $0.18 \mathrm{mmol}$ ), $\mathrm{CuCN}$ (48 mg, $0.54 \mathrm{mmol})$ and $\mathrm{Pd}\left(\mathrm{PPh}_{3}\right)_{4}(42 \mathrm{mg}, 20 \mathrm{~mol} \%)$ in $\mathrm{DMF}(5 \mathrm{~mL})$ gave the title compound as a yellow solid (66 mg, 73\%).

${ }^{1}$ H NMR $\left(500 \mathrm{MHz}, \mathrm{CDCl}_{3}\right) \delta 8.36(\mathrm{~d}, \mathrm{~J}=1.7 \mathrm{~Hz}, 1 \mathrm{H}), 8.34-8.30(\mathrm{~m}, 3 \mathrm{H})$, $8.20(\mathrm{~d}, \mathrm{~J}=1.3 \mathrm{~Hz}, 1 \mathrm{H}), 7.76(\mathrm{~d}, \mathrm{~J}=1.7 \mathrm{~Hz}, 1 \mathrm{H}), 7.72(\mathrm{~d}, \mathrm{~J}=1.7 \mathrm{~Hz}, 1 \mathrm{H}), 1.74$ $(\mathrm{s}, 9 \mathrm{H}), 1.57-1.49(\mathrm{~m}, 27 \mathrm{H}){ }^{13} \mathbf{C}$ NMR $\left(126 \mathrm{MHz}, \mathrm{CDCl}_{3}\right) \delta$ 154.4, 151.6, 149.3, 149.0, 135.3, 134.7, 131.3, 129.8, 129.2, 125.5, 124.3, 120.4, 119.9, 118.9, 118.7, 116.8, 36.2, 35.4, 35.01, 34.97, 31.36, 31.33, 31.29, 30.4 HRMS Found (ASAP+): $[\mathrm{M}+\mathrm{H}]^{+} 502.3481, \mathrm{C} 37 \mathrm{H} 44 \mathrm{~N}$ requires 502.3474
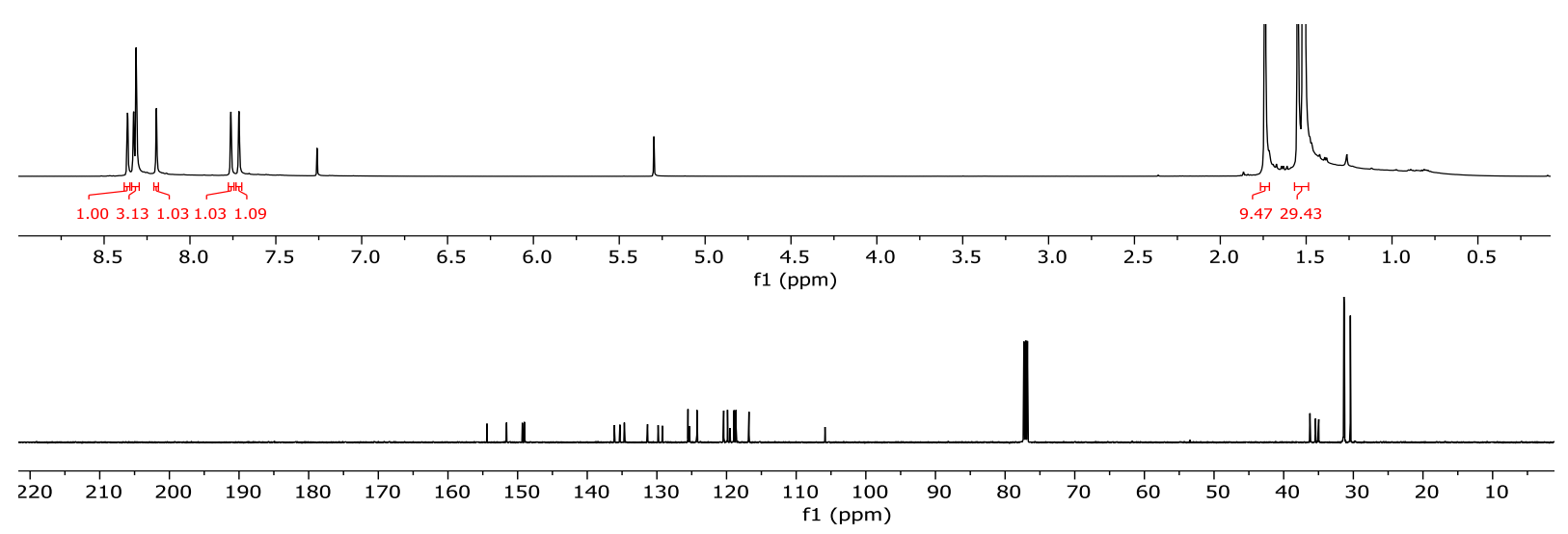

\section{3,(9/10)-Dibromo-2,5,8,11-terta-tert-butylperylene (16)}

A solution of 13 (180 mg, $0.38 \mathrm{mmol}, 1$ equiv.) and N-bromosuccinimide (200 $\mathrm{mg}, 1.13 \mathrm{mmol}, 3$ equiv.) in DMF ( $12 \mathrm{~mL}$ ) was stirred at room temperature for $16 \mathrm{~h}$. The mixture was diluted with water $(40 \mathrm{~mL})$ and extracted with DCM $(3 \times 30 \mathrm{~mL})$. The organic layers were collected, washed with water $(5 \times 50 \mathrm{~mL})$, dried with $\mathrm{Na}_{2} \mathrm{SO}_{4}$, and concentrated. Silica column chromatography, with hexanes as eluent, afforded the title compound as a yellow solid (180 mg, 75\%, 1:1.4 mixture of regioisomers).

${ }^{1}$ H NMR $\left(500 \mathrm{MHz}, \mathrm{CDCl}_{3}\right) \delta 8.45(\mathrm{t}, J=2.0 \mathrm{~Hz}, 4 \mathrm{H}), 8.40(\mathrm{~s}, 2 \mathrm{H}), 8.37(\mathrm{~s}$, $2 \mathrm{H}), 8.34(\mathrm{~d}, J=1.7 \mathrm{~Hz}, 2 \mathrm{H}), 8.30(\mathrm{~d}, J=1.7 \mathrm{~Hz}, 2 \mathrm{H}), 1.75(\mathrm{~d}, J=6.3 \mathrm{~Hz}, 36 \mathrm{H})$, $1.53(\mathrm{~d}, J=6.0 \mathrm{~Hz}, 36 \mathrm{H}){ }^{13} \mathbf{C} \mathbf{N M R}\left(126 \mathrm{MHz}, \mathrm{CDCl}_{3}\right) \delta 150.21,150.16,146.2$, 134.7, 130.6, 129.5, 126.7, 123.8, 123.7, 123.5, 119.9, 119.5, 119.1, 118.8, 37.9, 
37.8, 35.5, 35.4, 31.39, 31.36, 30.34, 30.31 HRMS Found (ASAP+): $[\mathrm{M}+\mathrm{H}]^{+}$

633.1737, $\mathrm{C}_{36} \mathrm{H}_{43} \mathrm{Br}_{2}$ requires 633.1732

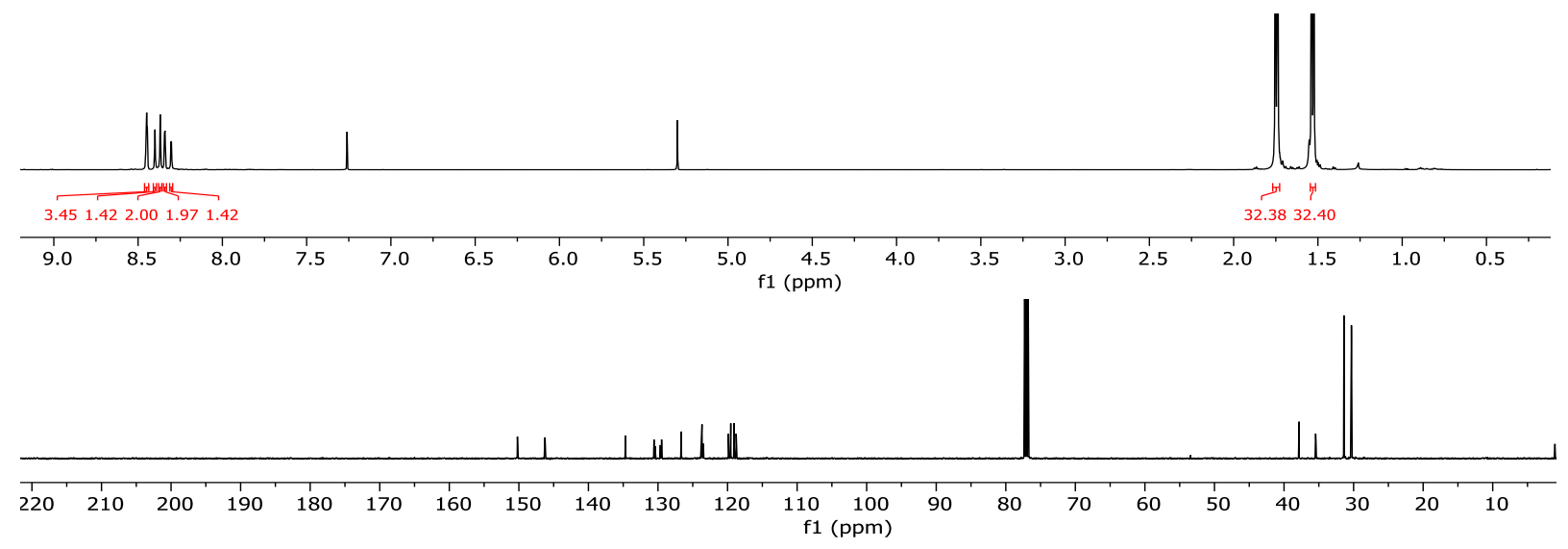

\section{3,(9/10)-Dicyano-2,5,8,11-terta-tert-butylperylene (17)}

Using general procedure I and dibromide $16(114 \mathrm{mg}, 0.18 \mathrm{mmol}), \mathrm{CuCN}$ (96 mg, $1.08 \mathrm{mmol})$ and $\mathrm{Pd}\left(\mathrm{PPh}_{3}\right)_{4}(84 \mathrm{mg}, 20 \mathrm{~mol} \%)$ in $\mathrm{DMF}(5 \mathrm{~mL})$ gave the title compound as a yellow solid (50 mg, 53\%, 1:1.4 mixture of regioisomers).

${ }^{1}$ H NMR $\left(500 \mathrm{MHz}, \mathrm{CDCl}_{3}\right) \delta 8.41(\mathrm{~d}, J=1.7 \mathrm{~Hz}, 2 \mathrm{H}), 8.40(\mathrm{~d}, J=1.6 \mathrm{~Hz}, 2 \mathrm{H})$, $8.38(\mathrm{~s}, 2 \mathrm{H}), 8.36(\mathrm{~s}, 2 \mathrm{H}), 8.30(\mathrm{~d}, J=1.6 \mathrm{~Hz}, 2 \mathrm{H}), 8.25(\mathrm{~d}, J=1.6 \mathrm{~Hz}, 2 \mathrm{H}), 1.73$

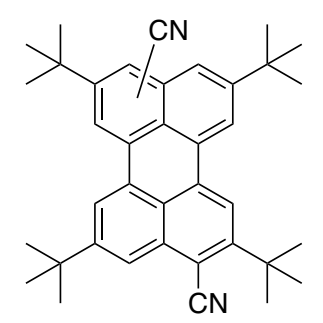
$(\mathrm{d}, J=5.0 \mathrm{~Hz}, 36 \mathrm{H}), 1.54(\mathrm{~d}, J=4.7 \mathrm{~Hz}, 36 \mathrm{H}) .{ }^{13} \mathbf{C ~ N M R}\left(126 \mathrm{MHz}, \mathrm{CDCl}_{3}\right) \delta$ 154.4, 152.12, 151.83, 135.82, 135.80, 133.4, 130.4, 125.0, 122.5, 121.3, 120.8, 119.8, 118.7, 117.9, 108.1, 36.32, 36.29, 35.5, 31.24, 31.22, 30.38, 30.35, 29.7 HRMS Found (ASAP+): $[\mathrm{M}+\mathrm{H}]^{+} 527.3430, \mathrm{C}_{38} \mathrm{H}_{43} \mathrm{~N}_{2}$ requires 527.3427

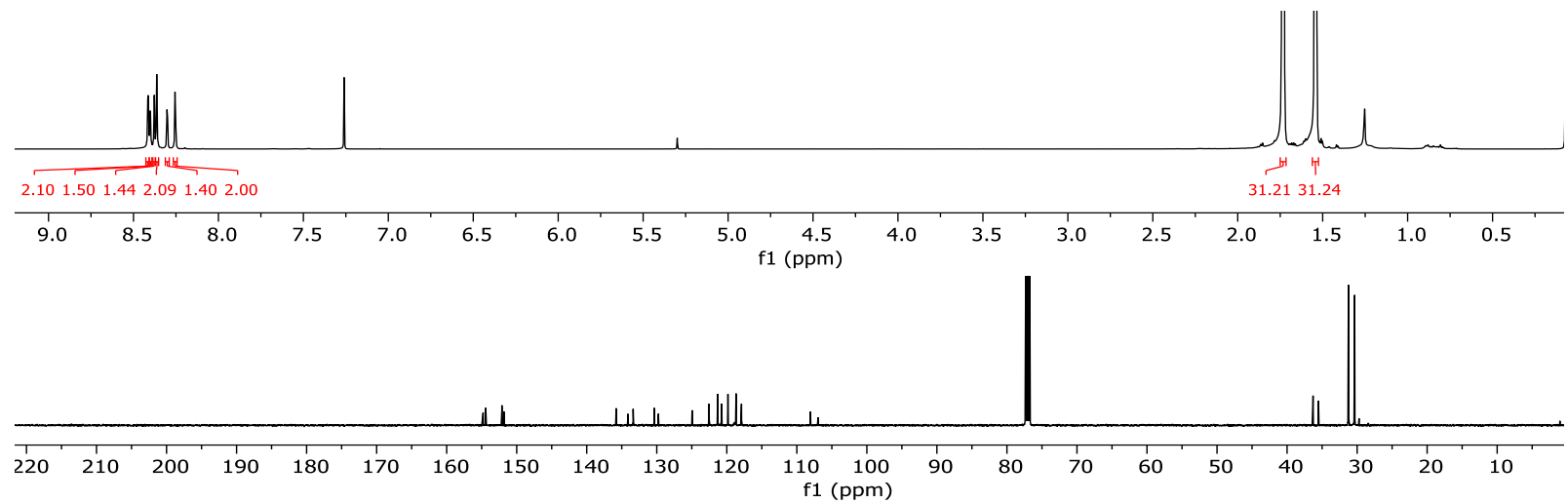




\subsubsection{Anthracenes}

9,10-Bis((triisopropylsilyl)ethynyl)anthracene-2-carbonitrile (18)

Using general procedure I and ((2-bromoanthracene-9,10-diyl)bis(ethyne-2,1diyl))bis(triisopropylsilane) (300 mg, $0.5 \mathrm{mmol}), \mathrm{CuCN}$ (131 mg, $1.5 \mathrm{mmol}$ ), $\mathrm{Pd}\left(\mathrm{PPh}_{3}\right)_{4}(113 \mathrm{mg}, 20 \mathrm{~mol} \%)$ in DMF $(15 \mathrm{~mL})$ gave the title compound as a yellow solid (193 mg, 70\%).

${ }^{1} \mathrm{H} \mathrm{NMR}\left(500 \mathrm{MHz}, \mathrm{CDCl}_{3}\right) \delta 9.07(\mathrm{~d}, J=1.6 \mathrm{~Hz}, 1 \mathrm{H}), 8.72(\mathrm{~d}, J=8.9 \mathrm{~Hz}, 1 \mathrm{H})$, $8.66(\mathrm{dd}, J=8.2,1.6 \mathrm{~Hz}, 2 \mathrm{H}), 7.76-7.65(\mathrm{~m}, 3 \mathrm{H}), 1.37-1.24(\mathrm{~m}, 42 \mathrm{H}){ }^{13} \mathbf{C}$

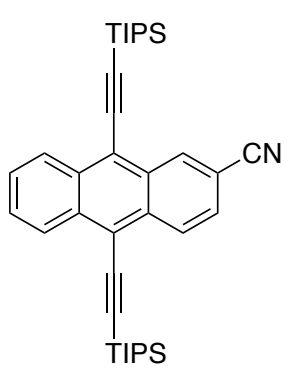
NMR $(125 \mathrm{MHz}, \mathrm{CDCl} 3) \delta 134.6,133.9,133.0,132.5,130.8,128.8,128.4$, 127.9, 127.6, 127.4, 126.1, 120.2, 119.3, 119.2, 110.2, 107.6, 106.5, 102.2, 101.9, 18.9, 11.5 HRMS Found (ESI+): $[\mathrm{M}+\mathrm{Na}]^{+} 586.3321, \mathrm{C}_{37} \mathrm{H}_{49} \mathrm{NNaSi}_{2}$ requires 586.3301

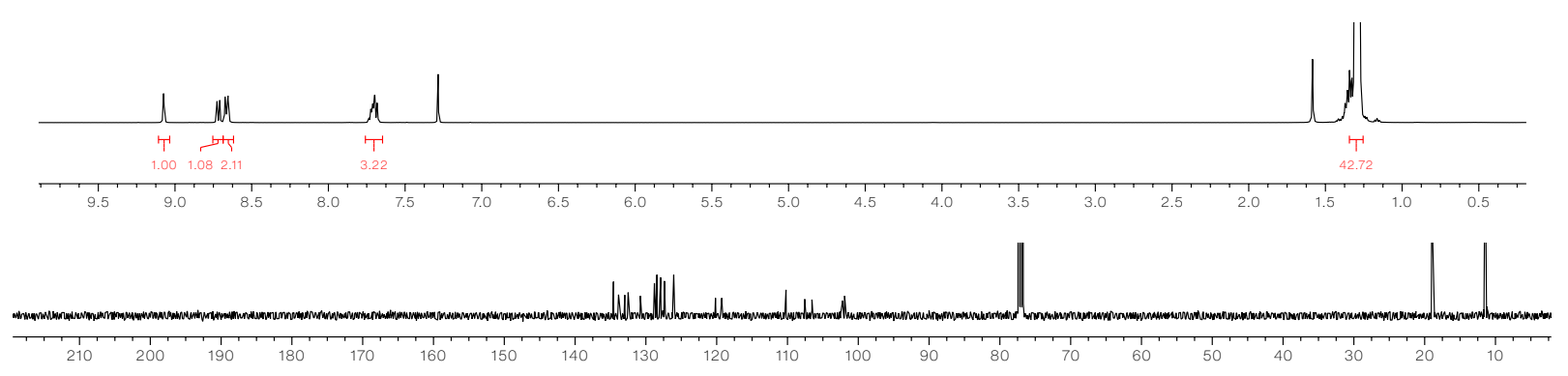

9,10-Bis((triisopropylsilyl)ethynyl)anthracene-2,6-dicarbonitrile (19)

Using general procedure I and ((2,6-dibromoanthracene-9,10-diyl)bis(ethyne2,1-diyl))bis(triisopropylsilane) (300 mg, $0.43 \mathrm{mmol}$ ), CuCN (231 mg, 2.6 $\mathrm{mmol}), \mathrm{Pd}\left(\mathrm{PPh}_{3}\right)_{4}(200 \mathrm{mg}, 40 \mathrm{~mol} \%)$ in $\mathrm{DMF}(15 \mathrm{~mL})$ gave the title compound as a yellow solid ( $245 \mathrm{mg}, 82 \%)$.

${ }^{1} \mathrm{H} \mathrm{NMR}\left(500 \mathrm{MHz}, \mathrm{CDCl}_{3}\right) \delta 9.07(\mathrm{~d}, J=1.6 \mathrm{~Hz}, 2 \mathrm{H}), 8.73(\mathrm{~d}, J=8.9 \mathrm{~Hz}, 2 \mathrm{H})$,<smiles>CC(C)C#Cc1ccc2c3ccc(C#N)cc3c3ccc(C#N)cc3c2c1</smiles>
$7.77(\mathrm{dd}, J=8.9,1.6 \mathrm{~Hz}, 2 \mathrm{H}), 1.39-1.25(\mathrm{~m}, 42 \mathrm{H}){ }^{13} \mathbf{C}$ NMR $(125 \mathrm{MHz}$, $\left.\mathrm{CDCl}_{3}\right) \delta 134.5,133.1,132.2,129.1,127.2,120.7,118.7,111.9,109.5,100.9$, 18.8, 11.4 HRMS Found (ASAP+): $[\mathrm{M}+\mathrm{H}]^{+} 589.3438, \mathrm{C}_{38} \mathrm{H}_{49} \mathrm{~N}_{2} \mathrm{Si}_{2}$ requires 589.3434

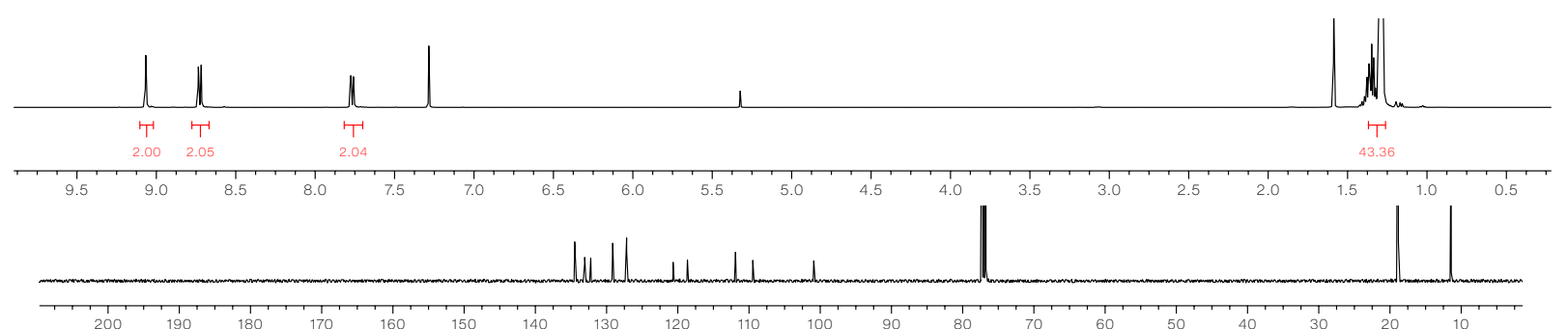


9,10-Bis ((triisopropylsilyl)ethynyl)anthracene-2,3-dicarbonitrile (20)

Using general procedure I and ((2,3-dibromoanthracene-9,10-diyl)bis(ethyne2,1-diyl))bis(triisopropylsilane) (300 mg, $0.43 \mathrm{mmol}$ ), CuCN (231 mg, 2.6 $\mathrm{mmol}), \mathrm{Pd}\left(\mathrm{PPh}_{3}\right)_{4}(200 \mathrm{mg}, 40 \mathrm{~mol} \%)$ in $\mathrm{DMF}(15 \mathrm{~mL})$ gave the title compound as a yellow solid (191 $\mathrm{mg}, 64 \%)$.

${ }^{1} \mathbf{H}$ NMR $\left(500 \mathrm{MHz}, \mathrm{CDCl}_{3}\right) \delta 9.15(\mathrm{~s}, 2 \mathrm{H}), 8.69(\mathrm{dd}, J=6.7,3.2 \mathrm{~Hz}, 2 \mathrm{H}), 7.81$

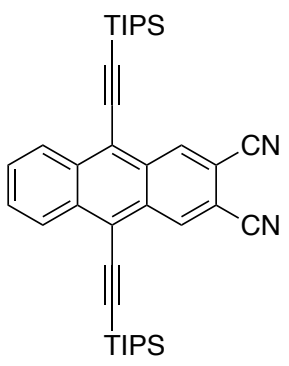
$(\mathrm{dd}, J=6.7,3.2 \mathrm{~Hz}, 2 \mathrm{H}), 1.39-1.24(\mathrm{~m}, 42 \mathrm{H}){ }^{13} \mathbf{C} \mathbf{~ N M R}\left(125 \mathrm{MHz}, \mathrm{CDCl}_{3}\right) \delta$ 136.6, 134.3, 130.7, 129.5, 127.8, 120.8, 116.0, 109.7, 109.3, 100.9, 18.8, 11.4 HRMS Found (ESI+): $[\mathrm{M}+\mathrm{Na}]^{+} 611.3244, \mathrm{C}_{38} \mathrm{H}_{48} \mathrm{~N}_{2} \mathrm{NaSi}_{2}$ requires 611.3254

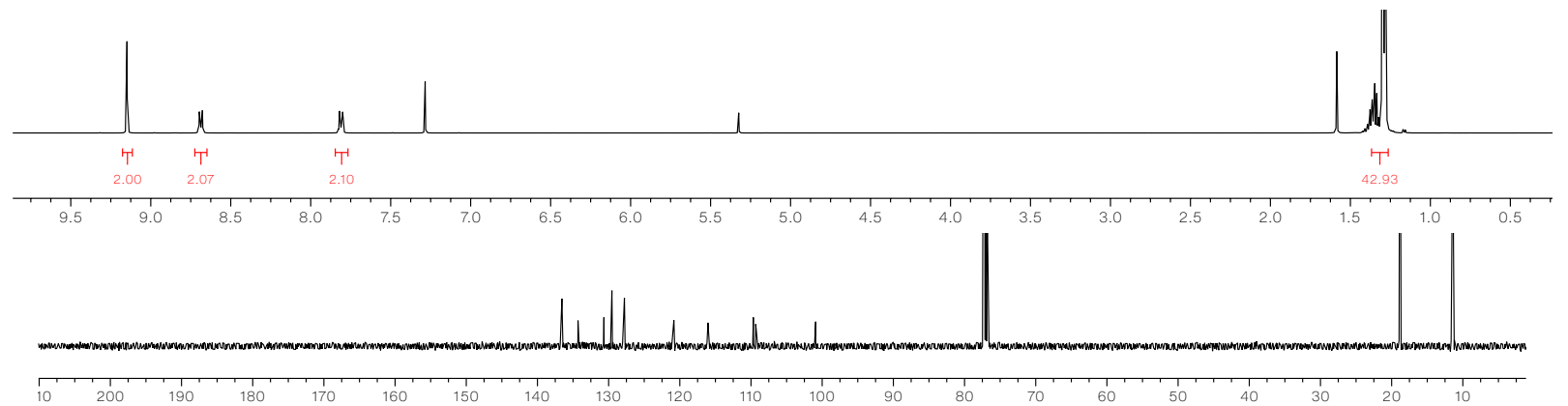




\subsection{METHODS}

UV-visible absorption spectra were obtained on a Technologies Cary 60 UV-Vis Spectrophotometer. Photoluminescence spectra were obtained on a Horiba FluoroMax 4 Spectrofluorometer. Fluorescence lifetimes were measured by time correlated single photon counting on a modified OB920 spectrometer (Edinburgh Analytical Instruments) using a pulsed LED with an emission maximum at $496 \mathrm{~nm}$ as excitation light source (PicoQuant).

\subsection{DATA}

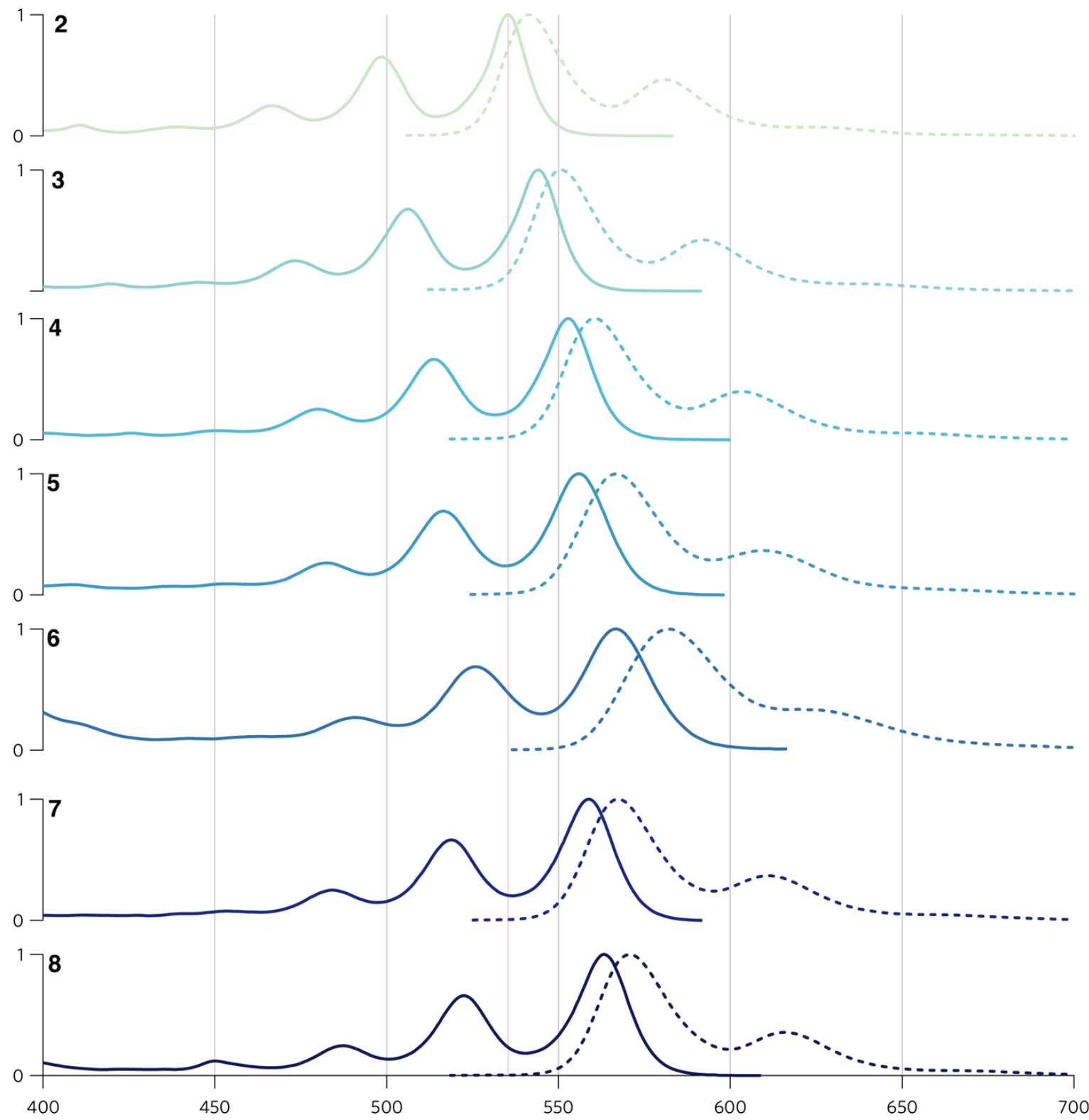

Figure S1 Normalised intensity steady-state absorption (solid lines) and emission (dashed lines) spectroscopy of the functionalised tetracene materials in dilute toluene $\left(10^{-6} \mathrm{M}\right)$. PL spectra were obtained by excitation at 375 $\mathrm{nm}$. 


\begin{tabular}{ccccccc}
\hline Annihilator & $\begin{array}{c}\text { Abs } \lambda_{\max } \\
(\mathrm{nm})^{a}\end{array}$ & $\begin{array}{c}E m \lambda_{\max } \\
(\mathrm{nm})^{a}\end{array}$ & $\mathrm{~S}_{0} \rightarrow \mathrm{S}_{1}(\mathrm{~nm})^{\mathrm{b}}$ & $\mathrm{S}_{0} \rightarrow \mathrm{S}_{1}(\mathrm{eV})^{\mathrm{b}}$ & $\varepsilon\left(\mathrm{M}^{-1} \mathrm{~cm}^{-1}\right)$ & PLQY (\%) \\
\hline $\mathbf{2}$ & 534 & 541 & 538 & 2.30 & 35846 & 86.8 \\
$\mathbf{3}$ & 544 & 551 & 547 & 2.26 & 39078 & 89.0 \\
$\mathbf{4}$ & 553 & 560 & 556 & 2.23 & 30459 & 87.9 \\
$\mathbf{5}$ & 556 & 567 & 561 & 2.21 & 26883 & 87.0 \\
$\mathbf{6}$ & 567 & 582 & 573 & 2.16 & 18752 & 90.5 \\
$\mathbf{7}$ & 559 & 567 & 563 & 2.20 & 29030 & 93.2 \\
$\mathbf{8}$ & 563 & 571 & 567 & 2.19 & 33860 & 64.0 \\
\hline
\end{tabular}

Table S1 Optical characteristics of the functionalised tetracene materials. (a) Maximum absorption/emission wavelength in toluene solution; (b) Estimated from crossing point of abs/emission spectra.

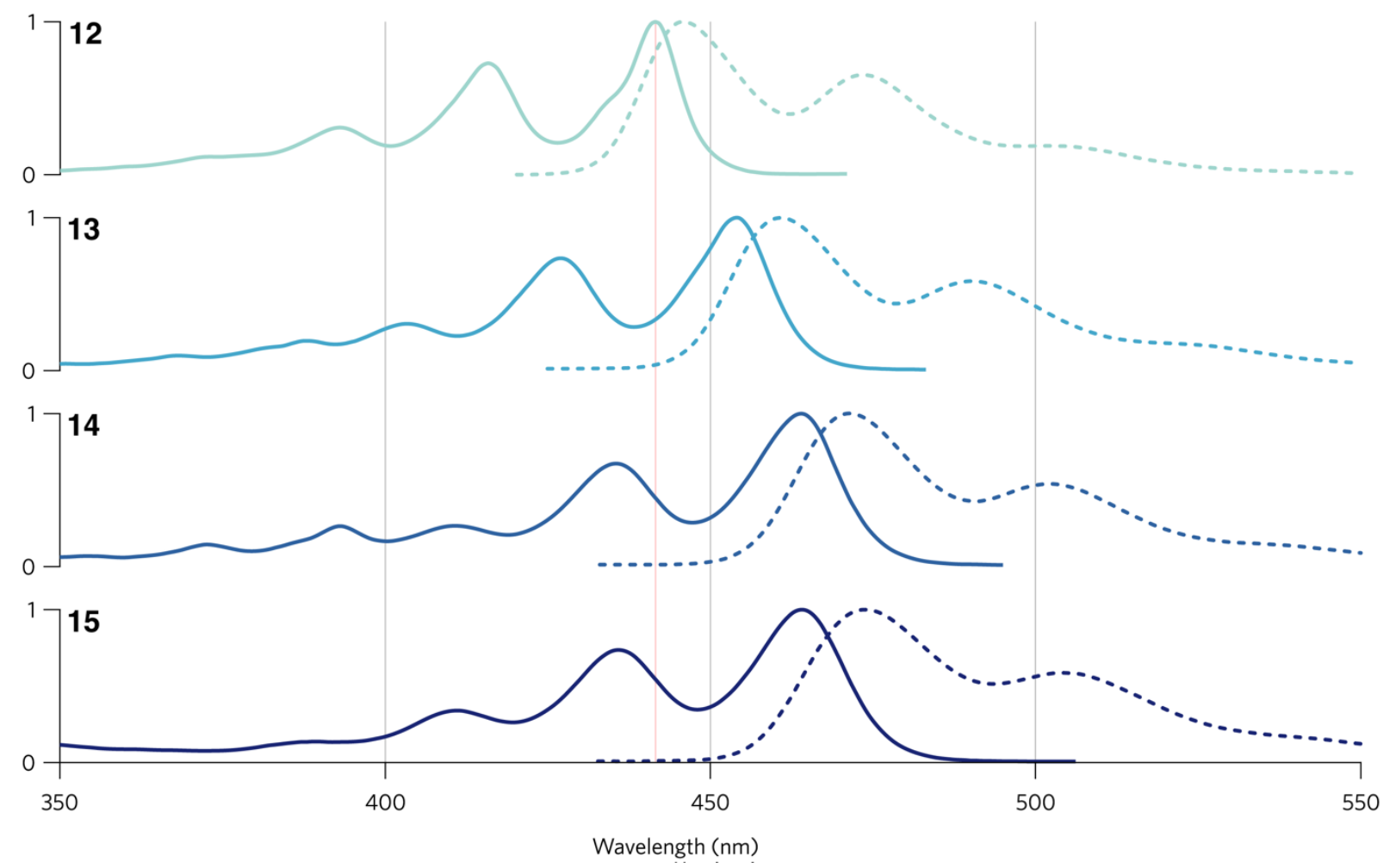

Figure S2 Normalised intensity steady-state absorption (solid lines) and emission (dashed lines) spectroscopy of the functionalised anthracene materials in dilute toluene $\left(10^{-6} \mathrm{M}\right)$. PL spectra were obtained by excitation at $300 \mathrm{~nm}$.

\begin{tabular}{ccccccc}
\hline Annihilator & $\begin{array}{c}\text { Abs } \lambda_{\max }(\mathrm{nm}) \\
\mathrm{a}\end{array}$ & $\begin{array}{c}\mathrm{Em} \lambda_{\max }(\mathrm{nm}) \\
\mathrm{a}\end{array}$ & $\mathrm{S}_{0} \rightarrow \mathrm{S}_{1}(\mathrm{~nm}) \mathrm{b}$ & $\mathrm{S}_{0} \rightarrow \mathrm{S}_{1}(\mathrm{eV}) \mathrm{b}$ & $\varepsilon\left(\mathrm{M}^{-1} \mathrm{~cm}^{-1}\right)$ & PLQY (\%) \\
\hline 12 & 441 & 446 & 443 & 2.80 & 50545 & 97.9 \\
13 & 454 & 461 & 457 & 2.71 & 43286 & 93.8 \\
14 & 464 & 472 & 467 & 2.66 & 39548 & 95.6 \\
15 & 464 & 474 & 468 & 2.65 & 54046 & 83.4 \\
\hline
\end{tabular}

Table S2 Optical characteristics of the functionalised anthracene materials. (a) Maximum absorption/emission wavelength in toluene solution; (b) Estimated from crossing point of abs/emission spectra. 


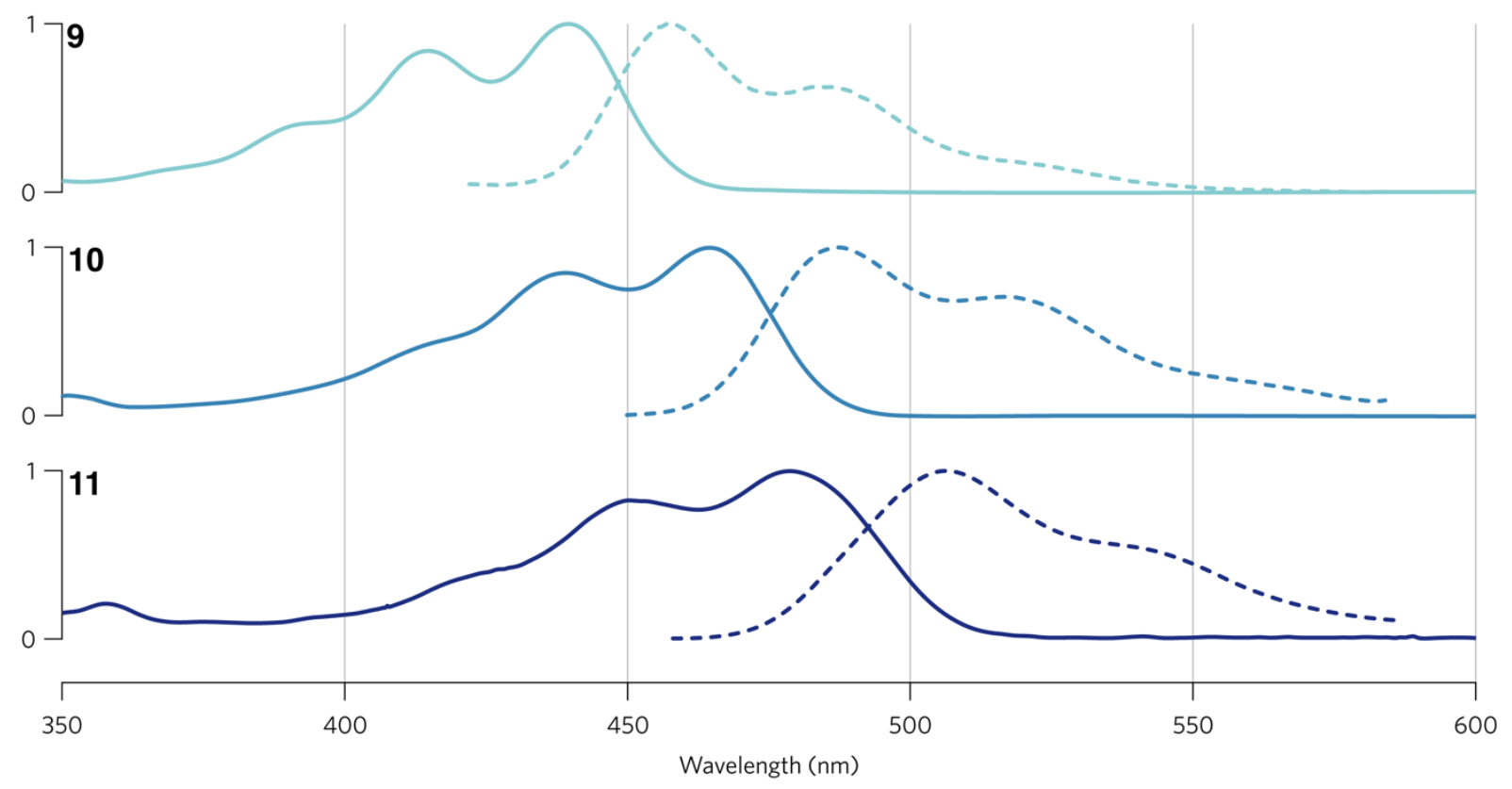

Figure S3 Normalised intensity steady-state absorption (solid lines) and emission (dashed lines) spectroscopy of the functionalised perylene materials in dilute toluene $\left(10^{-6} \mathrm{M}\right)$. PL spectra were obtained by excitation at 300 nm.

\begin{tabular}{ccccccc}
\hline Annihilator & $\begin{array}{c}A b s \lambda_{\max }(\mathrm{nm}) \\
\mathrm{a}\end{array}$ & $\begin{array}{c}\mathrm{Em} \lambda_{\max }(\mathrm{nm}) \\
\mathrm{a}\end{array}$ & $\mathrm{S}_{0} \rightarrow \mathrm{S}_{1}(\mathrm{~nm})^{\mathrm{b}}$ & $\mathrm{S}_{0} \rightarrow \mathrm{S}_{1}(\mathrm{eV})^{\mathrm{b}}$ & $\varepsilon\left(\mathrm{M}^{-1} \mathrm{~cm}^{-1}\right)$ & PLQY (\%) \\
\hline 9 & 440 & 457 & 448 & 2.77 & 25488 & 85.9 \\
10 & 467 & 490 & 478 & 2.59 & 55061 & 84.9 \\
11 & 480 & 507 & 493 & 2.52 & 24191 & 92.8 \\
\hline
\end{tabular}

Table S3 Optical characteristics of the functionalised perylene materials. (a) Maximum absorption/emission wavelength in toluene solution; (b) Estimated from crossing point of abs/emission spectra. 

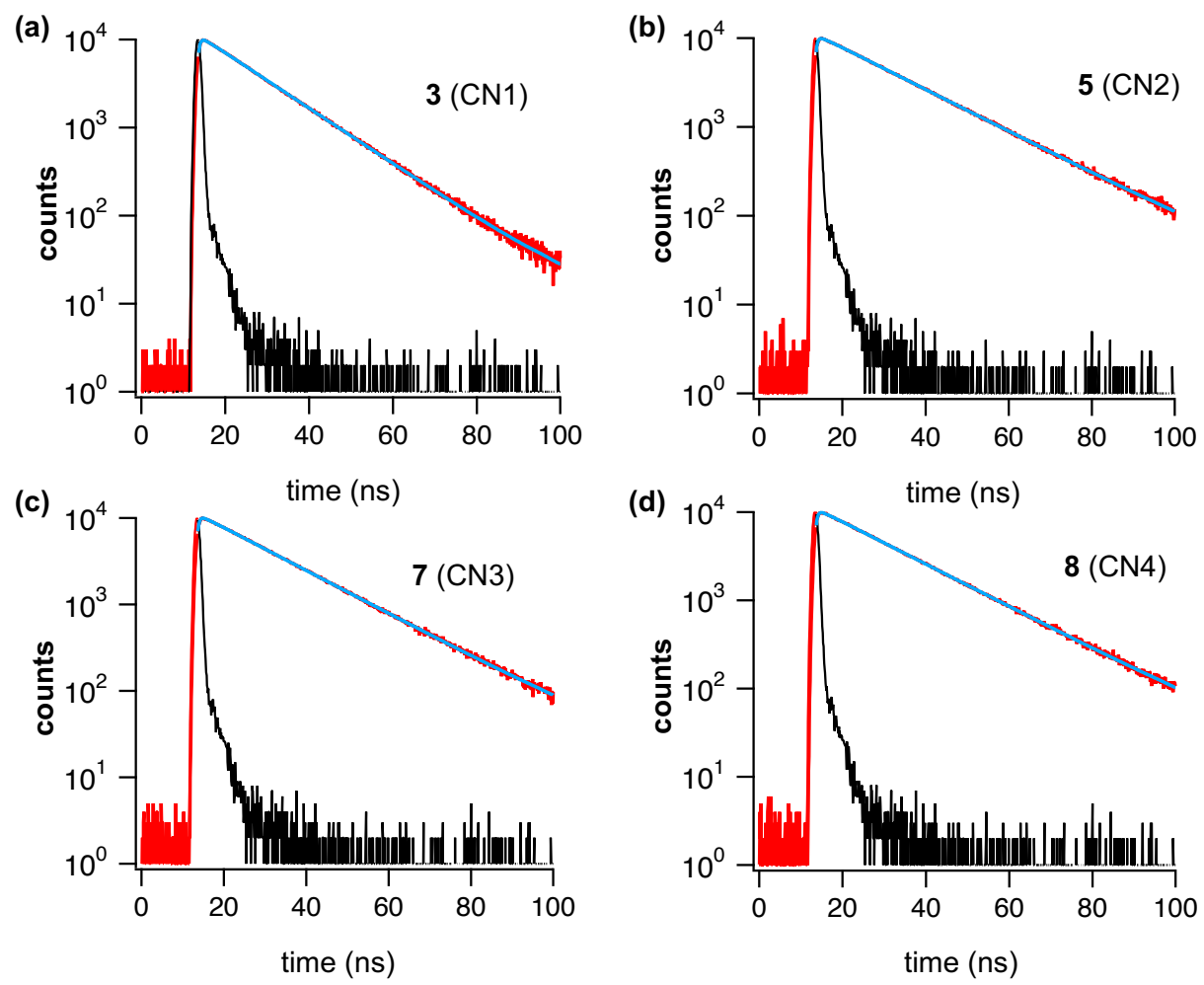

Figure S4 Fluorescence decay traces in Ar-saturated toluene (red) of TIPS-tetracene with increasing degrees of carbonitrile functionalization: 1, 2, 3 and 4 groups in compounds (a) 3, (b) 5, (c) 7, (d) 8 at 22 ${ }^{\circ} \mathrm{C}$ measured by time-correlated single photon counting $\left(\lambda_{\mathrm{ex}}=496 \mathrm{~nm}\right)$ monitored at $\lambda_{\mathrm{em}}=550 \mathrm{~nm}(\mathrm{a}), \lambda_{\mathrm{em}}$ $=567 \mathrm{~nm}(\mathrm{~b}, \mathrm{c})$ and $\lambda_{\mathrm{em}}=570 \mathrm{~nm}(\mathrm{~d})$. The instruments response function is shown in black. The singleexponential data fits with deconvolution of the instrument's response are shown in blue.

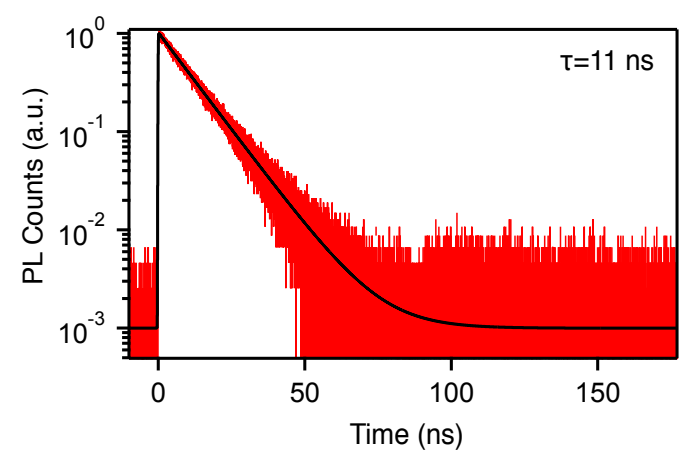

Figure S5 Fluorescence decay trace of TIPS-tetracene in degassed toluene. The measurement was taken using a Fianium super continuum laser running at $1 \mathrm{MHz}$. The output wavelength was determined using a set of increasing narrow interference filters to select a line centered at $488 \mathrm{~nm}$ with a bandwidth of $\sim 2 \mathrm{~nm}$. Excitation powers were kept in the few microwatt regime. Emission was collected with a parabolic mirror and focused onto the entrance slit of a iHR320 spectrometer. An output slit used to select the emission wavelength $(560 \mathrm{~nm})$ and the emission dynamics were recorded with an avalanche photodiode and photon counting electronics (PicoHarp). 


\begin{tabular}{cccc}
\hline Annihilator & No. of CN groups & $\tau_{\mathrm{f}}{ }^{\mathrm{Ar}}(\mathrm{ns})$ & $\tau_{\mathrm{f}}^{\mathrm{air}}(\mathrm{ns})$ \\
\hline $\mathbf{3}$ & 1 & $13.67 \pm 0.03$ & $10.96 \pm 0.03$ \\
$\mathbf{5}$ & 2 & $18.26 \pm 0.05$ & $13.88 \pm 0.04$ \\
$\mathbf{7}$ & 3 & $17.31 \pm 0.04$ & $14.03 \pm 0.04$ \\
$\mathbf{8}$ & 4 & $17.93 \pm 0.05$ & $15.21 \pm 0.04$ \\
\hline
\end{tabular}

Table S4 Fluorescence decay characteristics of tetracene derivatives in argon saturated $\left(\tau_{\mathrm{f}}^{\mathrm{Ar}}\right)$ and air saturated $\left(\tau_{f}^{\mathrm{air}}\right)$ toluene at $22{ }^{\circ} \mathrm{C}$ measured by time-correlated single photon counting $\left(\lambda_{\text {ex }}=496 \mathrm{~nm}\right)$ monitored at $\lambda_{\mathrm{em}}=550 \mathrm{~nm}(3), \lambda_{\mathrm{em}}=567 \mathrm{~nm}(5$ and 7$)$ and $\lambda_{\mathrm{em}}=570 \mathrm{~nm}(\mathbf{8})$. 


\subsection{METHODS}

Samples of upconversion systems were prepared in anhydrous degassed toluene in a $\mathrm{N}_{2}$ glovebox. Optical experiments were conducted under $\mathrm{N}_{2}$ in borosilicate cuvettes with a path length of $10 \mathrm{~mm}$. Photoluminescence (PL) was measured in a Labsphere QE Integrating Sphere using an Ocean Optics QEPro Spectrometer. All optical equipment was calibrated against a calibrated Newport photodetector.

\begin{tabular}{cccc}
\hline Annihilator PAH core & Sensitizer & UCPL probe $\lambda(\mathrm{nm})$ & PL probe $\lambda(\mathrm{nm})$ \\
\hline $\begin{array}{c}\text { Tetracene } \\
\begin{array}{c}\text { Anthracene } \\
\text { Perylene }\end{array}\end{array}$ & PdPc & 730 & 470 \\
\hline
\end{tabular}

Table S5 Upconversion annihilator/sensitizer parings and probe wavelengths for the UC and PL experiments.
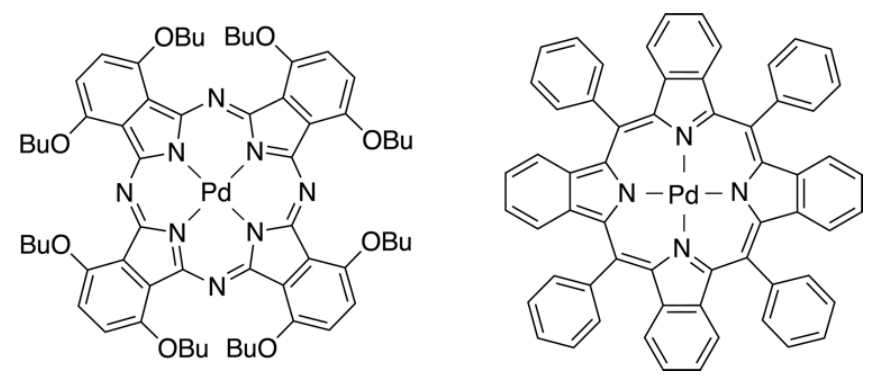

Figure S6 Sensitizers used in this study: PdPc (left) and PdTPTBP (right)

PdPc Palladium(II) 1,4,8,11,15,18,22,25-octabutoxy-29H,31H-phthalocyanine

PdTPTBP Palladium(II) meso-tetraphenyl-tetrabenzoporphyrin

\subsection{ANALYSIS OF UPCONVERSION}

\subsubsection{Measuring Upconversion}

The upconversion characteristics of each chromophore was determined following the method from de Mello et al. ${ }^{[5]}$ Briefly, a cuvette containing an upconverting system was placed centrally in an integrating sphere. Photoluminescence emission of the sample was recorded in three conditions: (a) cuvette in-sphere, in-beam; (b) cuvette in-sphere, out-of-beam; (c) cuvette out-of-sphere. Data was collected as an average of 40 scans over an integration time of $8 \mathrm{~ms}$. PdTPTBP samples were excited with a $500 \mathrm{~mW}$ M Series $635 \mathrm{~nm}$ from Dragon Lasers. PdPc samples were excited with a 730nm M series $1500 \mathrm{~mW}$ laser from Dragon Lasers. All quantum yield measurements were performed in the linear regime. Threshold measurements were determined by excitation from the same lasers and reducing the laser intensity with ND filters. The UC 
intensity was determined by the QE Pro Spectrometer; the laser intensity was determined from a calibrated Newport photodetector. The spot size was measured by scanning a razor blade across the focal plane.

\subsubsection{Optimising Rubrene}
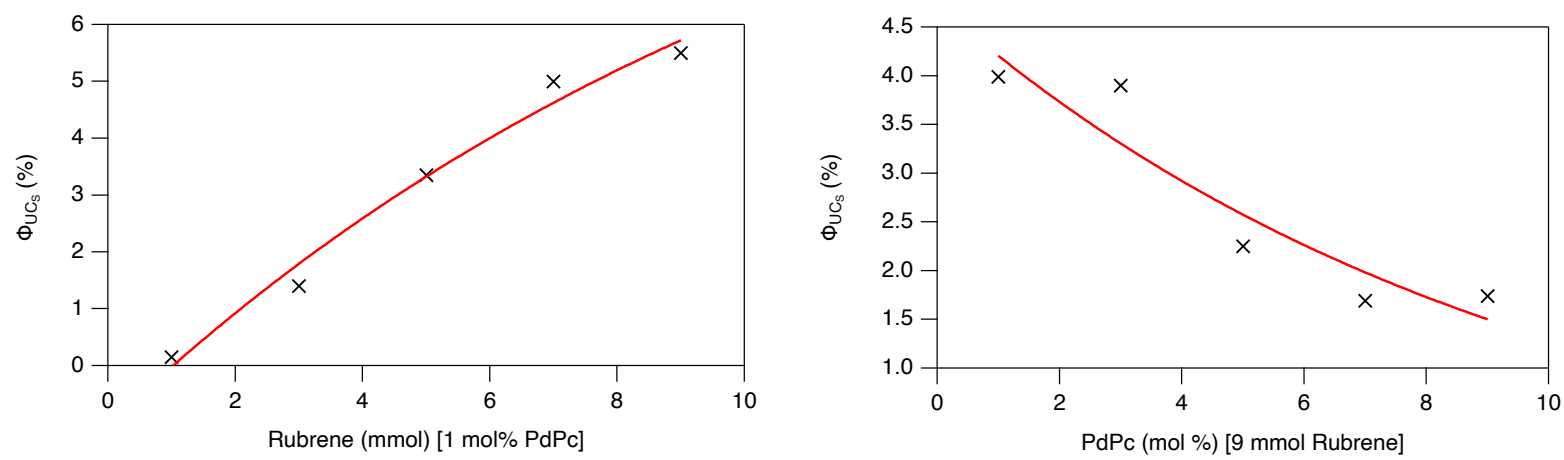

Figure S7 (Left) $\Phi_{\mathrm{UC}_{\mathrm{S}}}$ as a function of [Rubrene] with constant $\operatorname{PdPc}(1 \mathrm{~mol} \%)$. (Right) $\Phi_{\mathrm{UC}_{\mathrm{S}}}$ as a function of $[\mathrm{PdPc}]$ with constant rubrene $(9 \mathrm{mmol})$. The $\Phi_{\mathrm{UC}}$ is thus optimised at $9 \mathrm{mmol}$ rubrene $+1 \mathrm{~mol} \% \mathrm{PdPc}$ at $5.5 \%$. Note that $9 \mathrm{mM}$ rubrene in toluene is saturation, thus the limit of this experiment.

\subsubsection{Self-Absorption}

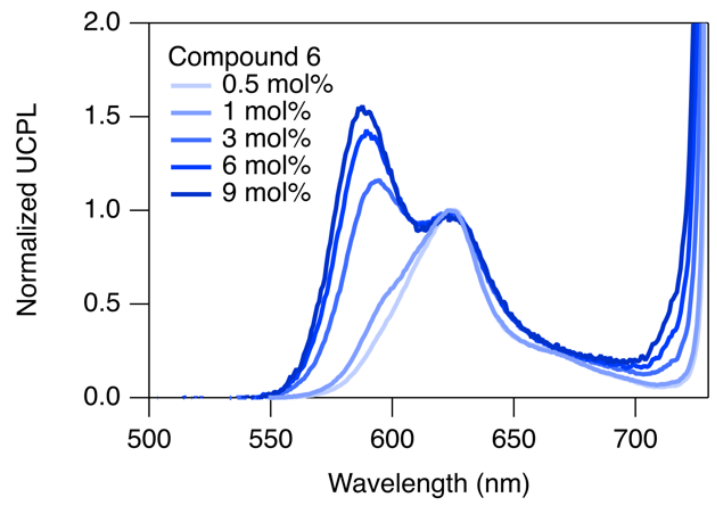

Figure S8 Due to the geometry of the experiment, the more concentrated samples exhibit less inner filtering. The concentrated solution has $81 \times$ the sensitizer concentration, and thus the emission is much closer to the front of the cuvette than in dilute solution, which upconverts throughout the length of the cuvette, and thus, has less overall path length to travel. This effect grows in with increasing sensitizer concentration. 


\subsubsection{Threshold Intensities}
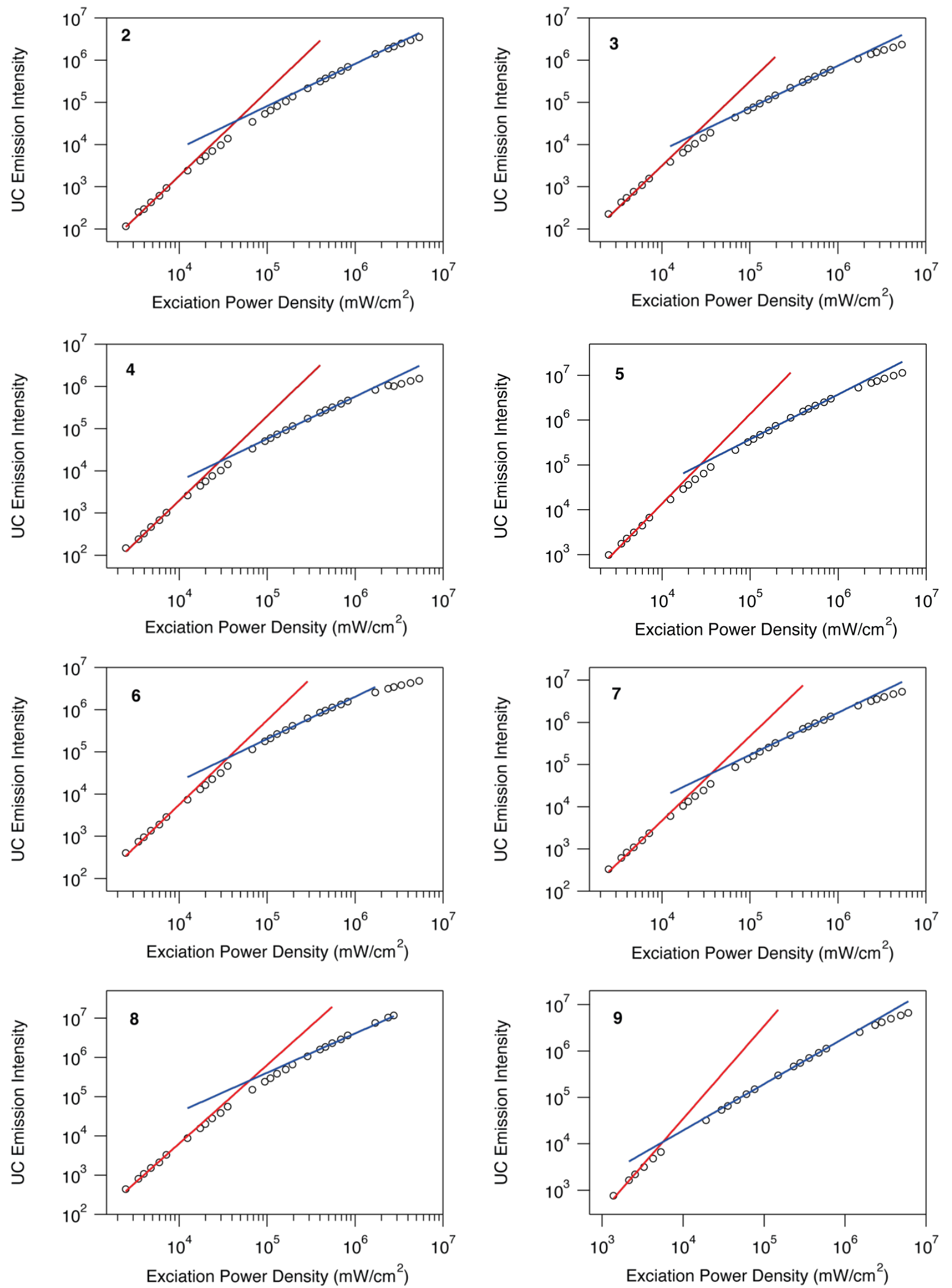

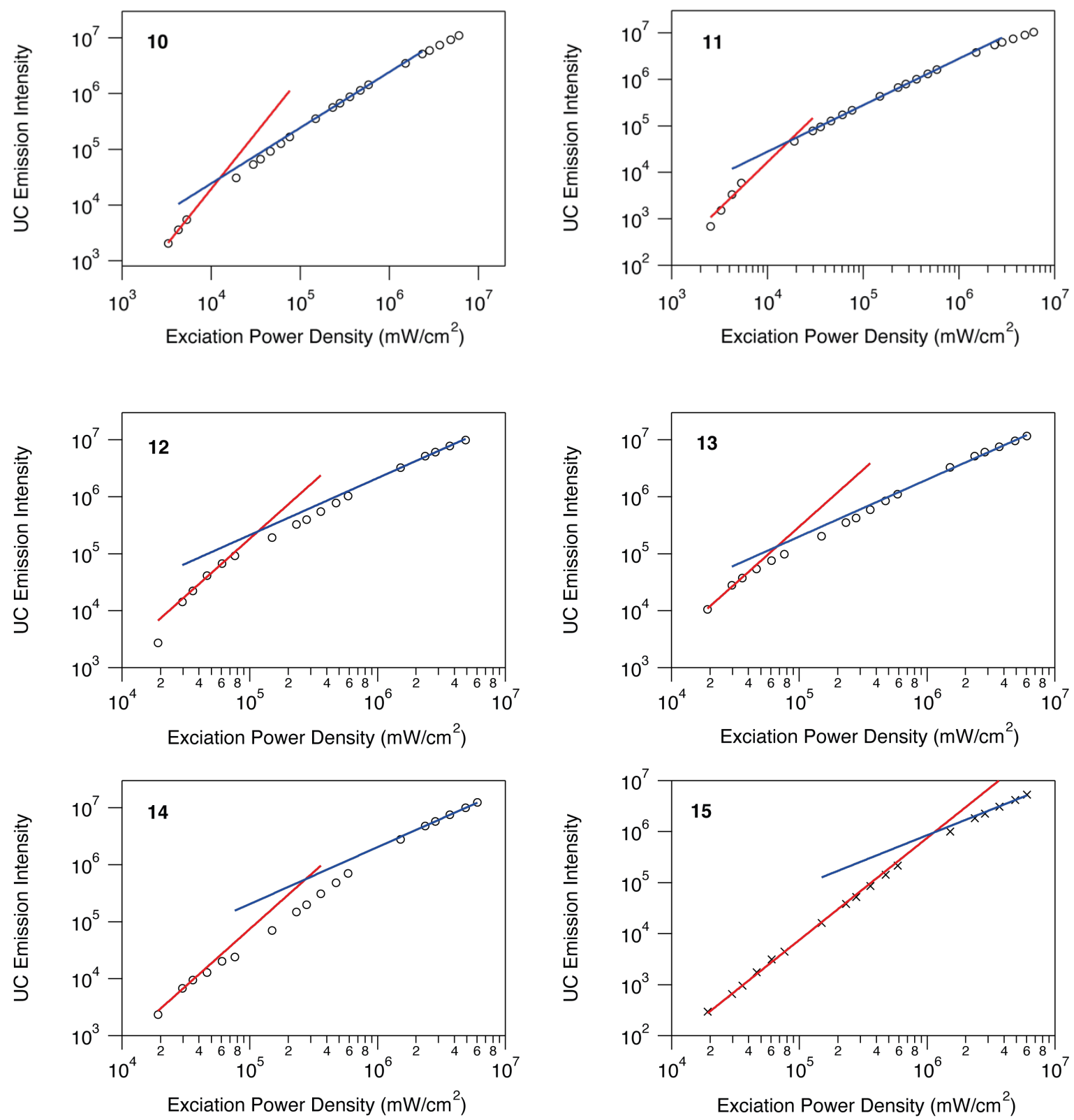

Table S6 Threshold intensities $\left(\mathrm{I}_{\mathrm{th}}\right)$ for the upconversion experiments $\left([\mathrm{An}]=1 \times 10^{-3} \mathrm{M}\right.$, $[$ Sens $]=1 \times 10^{-5}$ $\mathrm{M})$ in toluene using the excitation wavelengths as detailed in the section above.

\begin{tabular}{|c|c|}
\hline Compound & $\mathrm{I}_{\mathrm{th}}\left(\mathrm{mW} / \mathrm{cm}^{2}\right)$ \\
\hline 2 & 44,846 \\
\hline 3 & 23,345 \\
\hline 4 & 28,742 \\
\hline 5 & 27,217 \\
\hline 6 & 36,041 \\
\hline 7 & 36,022 \\
\hline 8 & 63,481 \\
\hline 9 & 5,592 \\
\hline 10 & 12,511 \\
\hline 11 & 16,721 \\
\hline
\end{tabular}




\begin{tabular}{|c|c|}
\hline 12 & 115,642 \\
\hline 13 & 66,794 \\
\hline 14 & 273,082 \\
\hline 15 & $1,126,711$ \\
\hline
\end{tabular}


Details of the transient absorption experiments have been described previously. ${ }^{[6]}$ Triplet energy transfer between the sensitizers and annihilators were studied using nanosecond transient absorption spectroscopy. To calculate the intrinsic lifetime of the porphyrin sensitizers $\left(\tau_{\mathrm{S}}\right)$, a $1 \times 10^{-5} \mathrm{M}$ solution of $\mathrm{PdPc}(\mathrm{PdTPTBP})$ in degassed (Ar) toluene was excited at $730(635) \mathrm{nm}$. The lifetime was calculated through a single exponential fitting to the decay of the excited state photoinduced absorption of the triplet states, (any singlet states would have decayed prior to the ns timescale). Triplet energy transfer was then probed in $1 \times 10^{-5} \mathrm{M} /$ $1 \times 10^{-3} \mathrm{M}$ mixtures of sensitizer/annihilator and $\tau_{\mathrm{S}+\mathrm{A}}$ calculated in identical experiments to $\tau_{\mathrm{S}}$. The quantum yield of triplet energy transfer $\left(\phi_{\mathrm{TET}}\right)$ was then calculated as

$$
\phi_{\mathrm{TET}}=1-\frac{\tau_{\mathrm{S}+\mathrm{A}}}{\tau_{\mathrm{S}}}
$$

Thus, the quantum yield of triplet-triplet annihilation $\left(\phi_{\mathrm{TTA}}\right)$ is given by

$$
\phi_{\mathrm{TTA}}=\frac{\Phi_{\mathrm{UC}}}{\phi_{\mathrm{ISC}} \phi_{\mathrm{TET}}}
$$

As we are only concerned with comparisons within chromophore families, we ignore contributions from

\begin{tabular}{|c|c|c|c|c|c|c|}
\hline $\begin{array}{l}\text { Annihilator } \\
\text { (A) }\end{array}$ & $\tau_{\mathrm{s}}(\mu \mathrm{s})$ & $\tau_{\mathrm{S}+\mathrm{A}}(\mu \mathrm{s})$ & $\phi_{\mathrm{TET}}$ & $\Phi_{\mathrm{UC}_{\mathrm{S}}}$ & $\phi_{\mathrm{TTA}^{\mathrm{a}}}{ }^{\mathrm{a}}$ & TTA $(\%)^{\mathrm{a}}$ \\
\hline 2 & \multirow{7}{*}{3.0} & 1.087 & 0.64 & 0.00135 & 0.00211 & 0.21 \\
\hline 3 & & 0.961 & 0.68 & 0.00205 & 0.00301 & 0.30 \\
\hline 4 & & 0.949 & 0.68 & 0.0018 & 0.00263 & 0.26 \\
\hline 5 & & 0.898 & 0.70 & 0.0155 & 0.02212 & 2.2 \\
\hline 6 & & 0.916 & 0.69 & 0.0345 & 0.04966 & 5.0 \\
\hline 7 & & 1.143 & 0.62 & 0.0065 & 0.01050 & 1.1 \\
\hline 8 & & 0.552 & 0.82 & 0.0135 & 0.01654 & 1.7 \\
\hline 9 & \multirow{7}{*}{101} & 3.61 & 0.96 & 0.056 & 0.05807 & 5.8 \\
\hline 10 & & 2.14 & 0.98 & 0.075 & 0.07662 & 7.7 \\
\hline 11 & & 2.62 & 0.97 & 0.084 & 0.08623 & 8.6 \\
\hline 12 & & 1.38 & 0.99 & 0.1275 & 0.12926 & 12.9 \\
\hline 13 & & 1.32 & 0.99 & 0.116 & 0.11753 & 11.8 \\
\hline 14 & & 1.16 & 0.99 & 0.1385 & 0.14011 & 14.0 \\
\hline 15 & & 1.17 & 0.99 & 0.0965 & 0.09763 & 9.8 \\
\hline
\end{tabular}
ISC, which are constant within each family.

Table S7 Triplet state lifetimes and corresponding quantum yields of excited state dynamics; (a) QY limit is $50 \%$ 


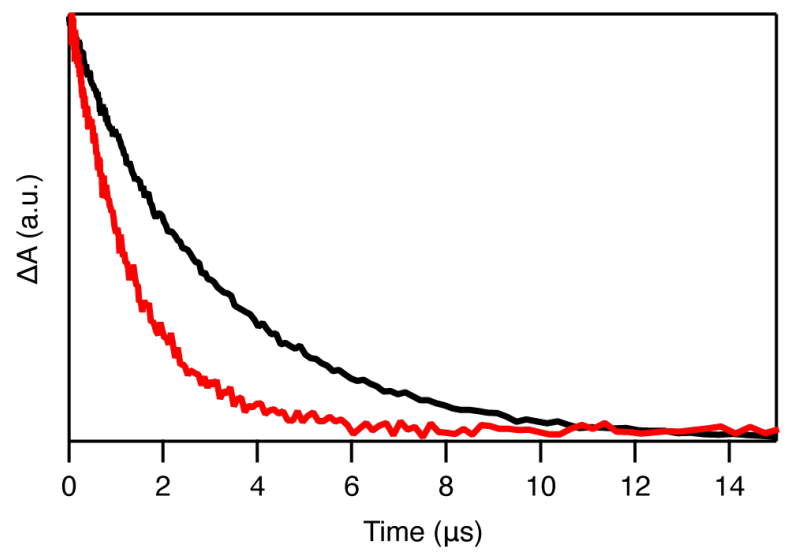

Figure S9 Decay of the photoinduced absorption of the triplet exciton of PdPc (at $615 \mathrm{~nm}$ ). The intrinsic lifetime is shown in black $\left(\tau_{\mathrm{S}}\right)$ and the same kinetic in the presence of tetracene 6 in red $\left(\tau_{\mathrm{S}+\mathrm{A}}\right)$ demonstrating the transfer of the triplet exciton from PdPc to 6.

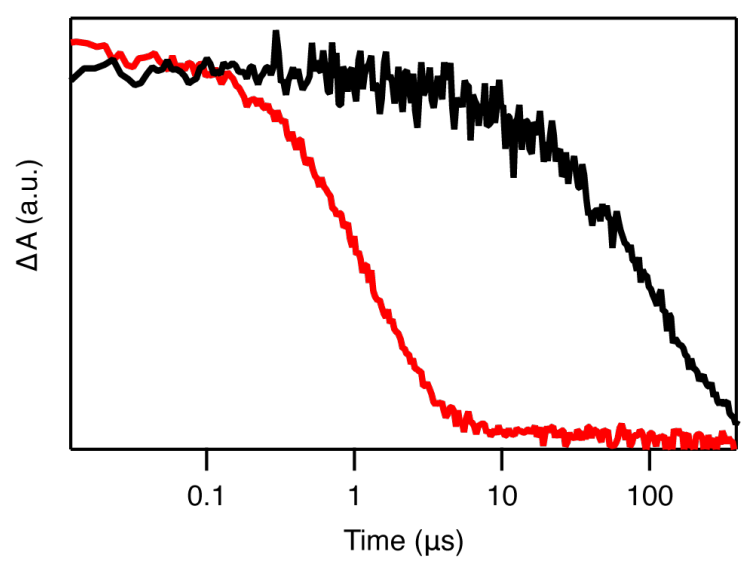

Figure S10 Decay of the photoinduced absorption of the triplet exciton of PdTPTBP (at $520 \mathrm{~nm}$ ). The intrinsic lifetime is shown in black $\left(\tau_{\mathrm{S}}\right)$ and the same kinetic in the presence of TIPS-anthracene $\mathbf{1 2}$ in red $\left(\tau_{\mathrm{S}+\mathrm{A}}\right)$ demonstrating the extremely efficient transfer of the triplet exciton from PdTPTBP to 12. 
All TDDFT calculations use the free ORCA package, version 4.2.0; density matrices are then processed and read into VMD to plot.

Figure S11 Plots of the difference in electron density in the excited and ground singlet states of the functionalized tetracenes. Red regions indicate locations of lower electron density in the excited state compared to the ground state, whilst blue regions indicate higher electron density in the excited state.

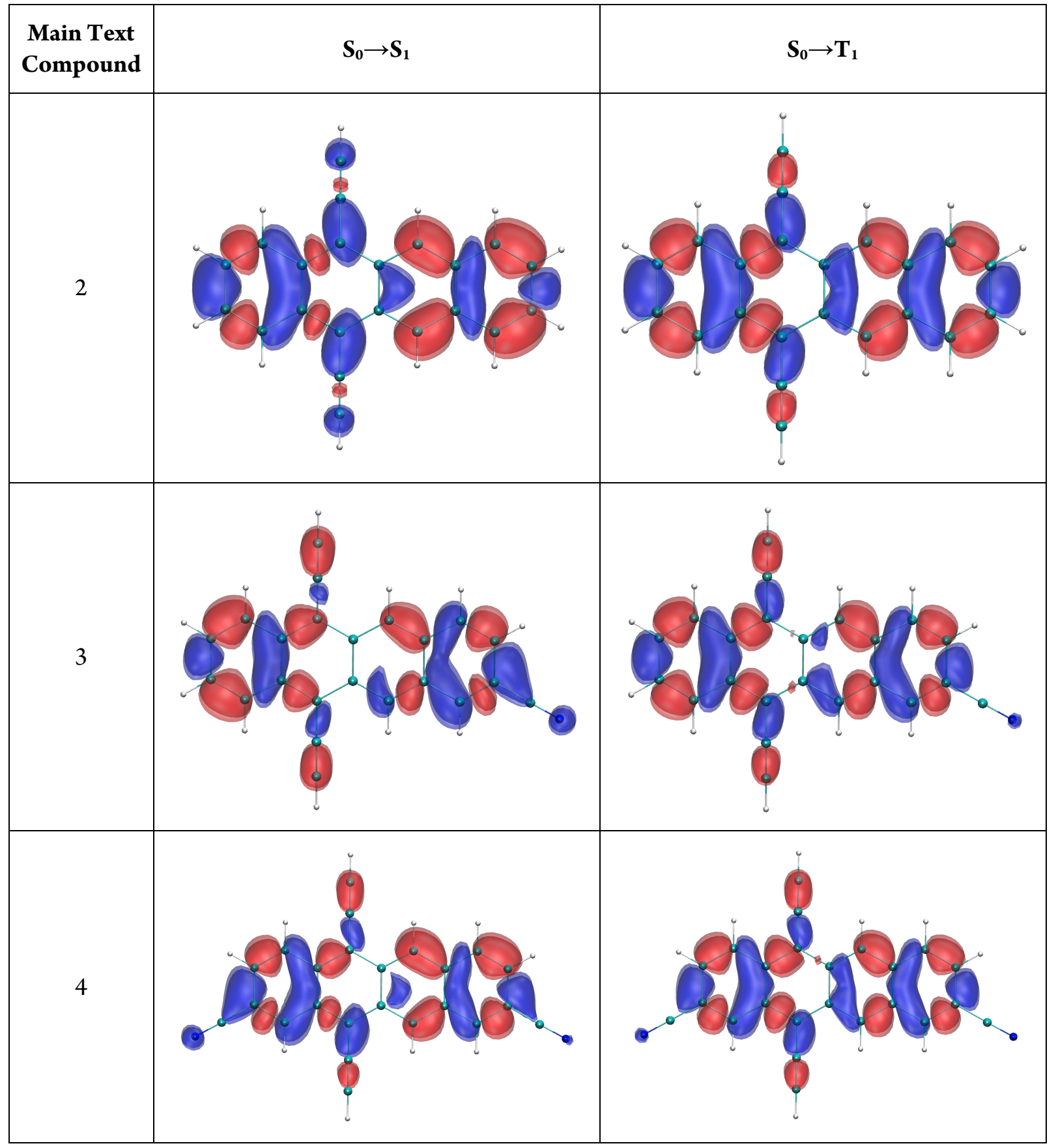




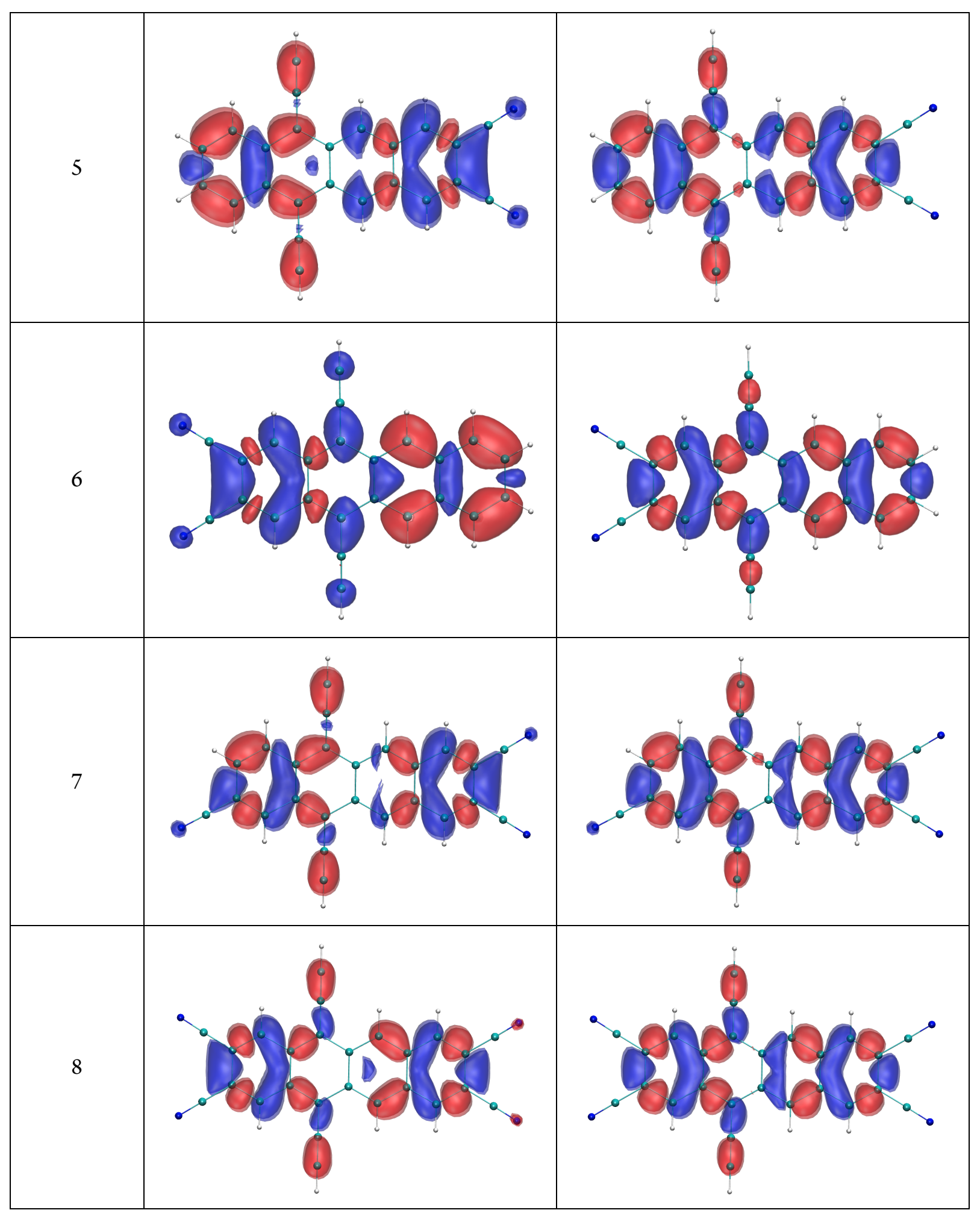


3. 16139565115203 0.69737201840144 3. 17461278996705 1. 92448975874338 1.95471445917021 4.37409539719782 1. 01812948911902 5. 31036623416919 3. 14612651253642 4. 39495883173781 5.61435012417576

5.60125176195899

4. 36645997407699

3. 18115891288729

6.55144679635528

6. 53150312852316

4.37999125003262

2. 24268928240527

$\odot .69602405932817$

1.94381247093309

1. 90318870870588

$\odot .71974947919774$

$-\odot .51059255004795$

$-\odot .51929977341359$

2. 83922778773385

๑.71294204510806

$-1.44420478901271$

$-1.45452955094153$

4.39180695648324

$-0.53318141020740$

5.43611988186670

6.35695694475832

$-1.57406045376124$

$-2.49192125244665$
2. 16696413534484

2. 16708780469315

2. 16633821895661

2. 16639977241276

2.16580390490641

2. 16568491736575

2. 16573981547193

2. 16553028064286

2.16520506444526

2. 16514301347279

2. 16455394623104

2. 16409343102444

2. 16415583948153

2. 16467611675993

2. 16451541405619

2. 16368944651574

2. 16379893607549

2. 16473246440969

2. 16762769008042

2. 16756112433244

2. 16819112124500

2. 16891211402289

2. 16898496621302

2. 16832912274212

2. 16797958724562

2. 16944905174444

2. 16958106537975

2. 16822730822595

2. 16724499165651

2. 16748875852500

2. 16697052834848

2. 16609826674795

2. 16732306735155

2. 16656056577805

\section{Compound $2 / \mathrm{S}_{1}$}

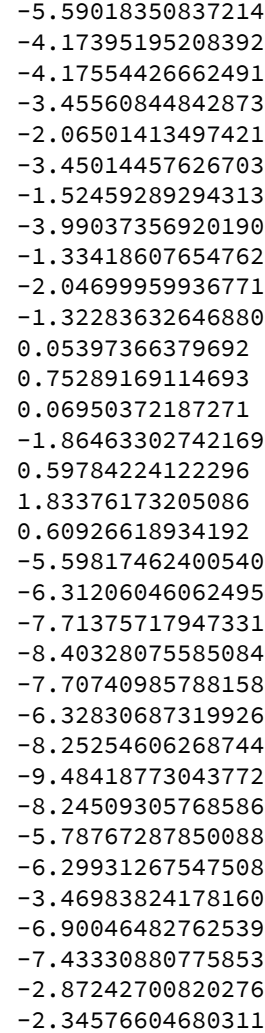

3.16006583121129

๑. 70811165201908

3. 17302253211180

1.92674375151720

1. 96159055926266

4. 35989399748361

1.02533012432338

5. 29623939992105

3. 15963769930157

4.39386887750872

5.59659042297049

5.59089556676069

4. 38056827594677

3. 18535714946610

6.53295763043175

6.52477326650716

4. 38469147903199

2. 24791583121635

$\odot .69655190405668$

1. 93252557536452

1.89884973993104

0.70457803691634

$-\odot .50044019256808$

$-0.50045299177130$

2.83459926930394

๑. 70764066834775

$-1.43794160624163$

$-1.43519686334166$

4. 38278091712293

$-\odot .51742569017718$

5. 42154808912665

6. 33977093349357

$-1.55835778829904$

$-2.48013312136247$
2. 16705053908941

2.16714598310143

2. 16647474084624

2. 16654298819589

2. 16598901167007

2. 16584463312131

2. 16607378471502

2. 16578353825171

2. 16534846130600

2.16524979130525

2.16458059271356

2. 16401795317401

2. 16412012330777

2. 16477730261110

2. 16450471196082

2. 16349563462143

2. 16367738191836

2. 16485706126999

2. 16776445569471

2. 16769545822017

2. 16829561436816

2. 16893928256491

2. 16899923278179

2. 16841456273728

2. 16825437022458

2. 16940012763037

2. 16950194077961

2. 16846473193401

2. 16697275578786

2. 16718109900582

2.16651880772003

2. 16574338984225

2. 16682462037053

2. 16613716906415 
3. 17225186038095 $\odot .69391727727515$

3. 18424439188339

1.92883144365865

1.96013070622458

4.36962444231993

1. 02454100169955

5.30750776047254

3. 17145510736266

4. 40545430777303

5.61133654335687

5.60693801998294

4. 39418988153997

3. 19447981150164

6. 54872589957887

6.54238216949673

4.39716088802163

2. 25615025333194

$\odot .68667595096585$

1.91885582657762

1.88978386263579

$\odot .68773609786100$

$-\odot .51435034753596$

$-\odot .50985969320743$

2. 82592735494536

$\odot .69555271348678$

$-1.45508695153828$

$-1.44565491502247$

4.38520980634407

$-\odot .52700261344583$

5.42716342715889

6. 34227151905291

$-1.57802360532677$

$-2.50136927191880$
2. 16380205542136

2.16394350436120

2. 16485479108595

2. 16492464824665

2.16557578615604

2. 16544385320867

2. 16537984082674

2. 16514238534816

2. 16648872662208

2. 16642213358998

2. 16733643759492

2. 16835804568785

2. 16842292647839

2. 16746578708454

2. 16726164915152

2. 16910563886690

2. 16922038359763

2. 16749105675011

2. 16784373366277

2.16777327409137

2. 17182451593419

2. 17599491207120

2. 17606587396623

2. 17196445741386

2. 17170183757545

2. 17918706263907

2. 17931376848692

2. 17194777333685

2. 15934192638786

2. 15962738914180

2.15491077835934

2. 15032145050801

2. 15532716232960

2. 15085628592236

\section{Compound 3 / $\mathrm{S}_{0}$}

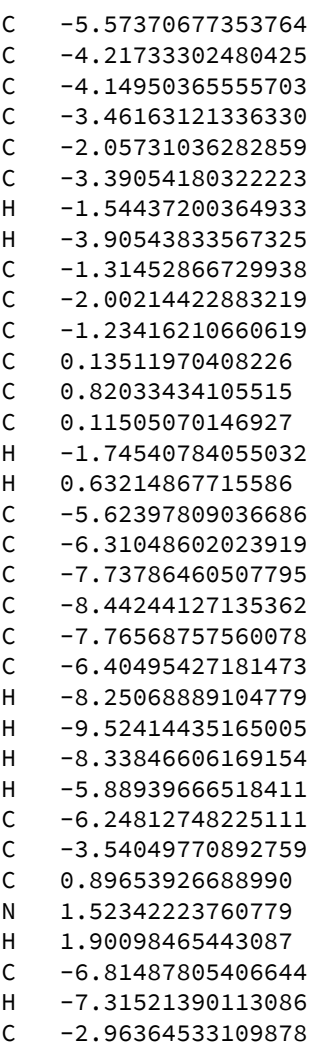

$-2.96364533109878$
3. 15706508947512 $\odot .65379314961932$

3. 12909022634347

1. 86026676315096

1. 85111469369299

4. 30862568251001

0.89945482173806

5.25922358553194

3. 02123590205611

4. 28820542934078

5.48648581304993

5.44103193160947

4. 17995674702548

3. 02003668223968

6.43969387309552

2. 06916949826668

$\odot .69203370306931$

1.95893968961551

1.95815353335542

๑. 79329628460391

$-\odot .45565508857403$

$-\odot .50246249030483$

2. 90893475083472

$\odot .81497565140613$

$-1.37355753329923$

$-1.45174514444373$

4.40721799135919

$-0.59528167302236$

6. 64937506946770

7.61736457959572

4.17378971908727

5.46903934434659

6.40556558184835

$-1.65166807423807$

$-2.58390194670660$
2. 16757898250753

2. 16822107975828

2. 16294219021942

2. 16326659232677

2. 15863241140057

2. $15800642 \odot 46528$

2. 15893860272832

2. 15781413743716

2. 15371054006182

2. 15338817795952

2. 14830121354416

2. 14376292218045

2. 14408428613986

2. 14889146021252

2. 14803503797115

2. 14912303065915

2. 17262120456592

2. 17229320081557

2. 17681800029543

2. 18128838153563

2. 18161472484000

2. 17746001760821

2. 17662709721086

2. 18460588783505

2.18517820339330

2. 17777084015403

2. 16741496218400

2. 16870274792168

2. 13864733358184

2. 13435222297803

2. 14043966890563

2. 16700871420788

2. 16637902376371

2. 1688333525888

2. 16866271985715 


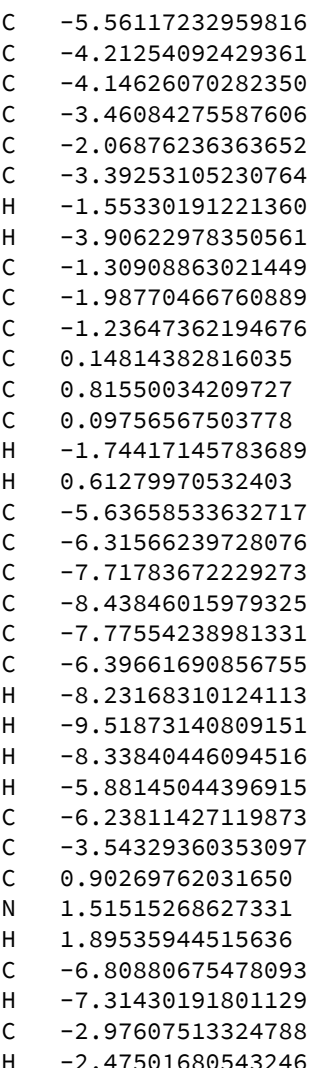

\section{Compound $3 / \mathrm{T}_{1}$}

3.15298049084057 $\odot .66867712004622$

3. 13116581186502 1.86399799084531 1.85932171267491 4. 29788292495375 $\odot .90953637800089$ 5.24861739663010 3.03580758911922 4. 29198227165880 5.47267848385634 5.42916665301254

4. 19245949696410

3. 02387142085139 6.42679647488381 2. 07330503038997

$\odot .69364300833263$ 1. 94726988496770

1. 95276365882501

$\odot .77720839478499$

$-0.44613885729955$

$-\odot .48352643940996$

2.90235882334981

$\odot .80914148269082$

$-1.36848546712146$

$-1.43243958519300$

4.39385844026841

$-\odot .57711654523773$

6.63971531101729

7.60983690983950

4. 17470990190961

5.44892226341672

6.38302115396693

$-1.63387628252829$

$-2.57024946642589$
2. 16743099312431

2. 16788907334254

2. 16281233363671

2. 16314260967581

2.1586140680920॰

2. 15805594227253

2. 15898646066755

2. 15773242027152

2. 15375426971457

2. 15332067131353

2. 14836306959531

2. 14381143182744

2. 14423613994698

2. 14912881271041

2. 14802589550932

2. 14944462537440

2. 17261970199053

2. 17216392426519

2. 17667589490760

2. 18152408365470

2. 18200151856277

2. 17755159611022

2. 17632761231062

2. 18499880215066

2. 18583702972019

2. 17789040295137

2. 16717645116259

2. 16825648098589

2. 13875064377914

2. 13456048602979

2. 14064094081489

2. 16677188573962

2. 16628012086627

2. 16835686096885

2. 16828213576888
3.16701517376255

$\odot .65335140133584$

3. 14137434167391

1.86632771634666

1. 85790855710934

4. 30643266441588

$\odot .90777420651792$

5.25911678489868

3. 04607519929465

4.30059127001552

5.48377801266688

5.44245297595153

4. 20223578058872

3. 03028722414367

6.43907442471084

2. 07867245370463

$\odot .68402076912508$

1.93441521972750

1.94252336050025

0.75911693445478

$-0.46110742392810$

$-0.49332088108620$

2. 89222227447329

$\odot .79548028214648$

$-1.38729983271976$

$-1.44315184358297$

4.39920271762071

$-0.58576616085051$

6.65554089114194

7.63384702316215

4. 18200658752368

5.45886275505865

6. 39018450892974

$-1.65156858384190$

$-2.58881294824552$
2.16737000171403

2. 16816201807505

2. 16275662249244

2. 16317017492903

2. 15868922588909

2. 15789616022061

2. 15902095418162

2. 15759998912686

2. 15368941060491

2. 15327317868235

2. 14834850839449

2. 14388492945878

2. 14430745397223

2. 14913094310098

2.14800306000210

2. 14944503661017

2. 17269417054081

2. 17229733649583

2. 17669812342307

2. 18139185591392

2. 18177876107792

2. 17746769238184

2. 17641537588625

2. 18471377284535

2. 18540472302632

2. 17778398781885

2. 16715014204851

2. 16871889410887

2. 13887243203801

2. 13469070866467

2. 14082296044245

2. 16659855777620

2. 16577651263417

2. 16882578141418

2. 16856593382274 


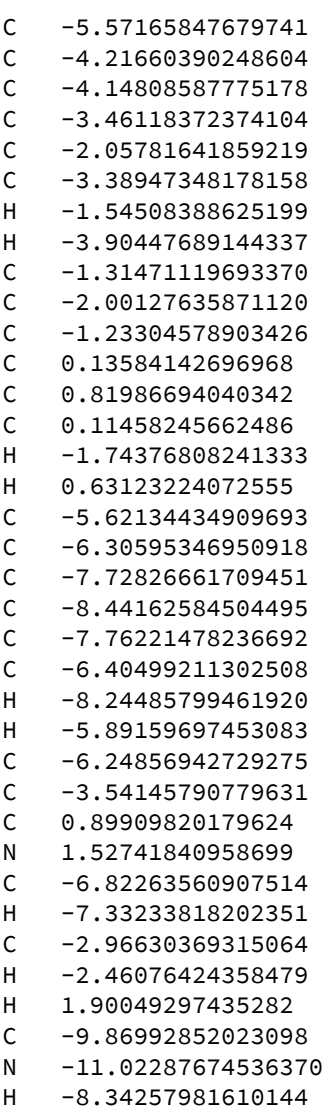

\section{Compound $4 / S_{1}$}

3. 16138303107519

$\odot .65333804708338$

3. 13088115258451

1. 86124630990122

1.85133002352267

4.31062235571446

$\odot .89965300593986$

5.26109596679521

3. 02184301373321

4.28932520162850

5.48771502495821

5.44066999104518

4. 17909871212070

3. 01917666003262

6.44109638960615

2. 06820862358743

$\odot .69355977181191$

1.96039948889222

1.96660763464172

$\odot .79731171012150$

$-0.46078284266827$

$-\odot .50036168403785$

2.91463200476554

$-1.45059694201035$

4.40909684013533

$-0.59635836375101$

6.64806925003269

7.61488895862726

5.46664200993289

6.39853704927979

$-1.65335783097257$

$-2.58729933257086$

4.17242535023048

$\odot .82465561272155$

0.83005030860167

$-1.37232130146404$
2. 16728344653627

2.16800005254324

2. 16269236871113

2. 16305323225819

2.15848604206088

2. 15779126426719

2. 15876307649317

2. 15752476735934

2. 15365497549324

2. 15329758154501

2. 14832805227017

2. 14394966974597

2. 14430083786305

2. 14898820873249

2. 14804217618922

2. 14925118916453

2. 17252107247589

2. 17215739523394

2. 17680324099082

2. 18154799568796

2. 18191117422206

2. $17754452 \odot 83286$

2. 17654496234416

2.17781757566734

2. 16692569820900

2. 16834440753560

2. 13898326190089

2. 13496726204075

2. 16649668630515

2. 16606999348052

2. 16842200353955

2. 16847882887485

2. 14078150396196

2. 18617195637190

2. 18993805020914

2. 18568911910069
3.15803274260240 $\odot .66866878301247$ 3.13191964519622 1.86597095728610 1.85921841806653 4. 29849788128081 $\odot .90859424520217$ 5. 24947226020725 3. 03610710636288 4. 29085002445099 5.47227470629696 5.42709340963319

4. 19073742537574

3. 02188149516017

6.42671562802048

2. 07131358745302

$\odot .69591072143590$ 1. 95099340296692

1.96194832364775

0.77997077338783

$-0.44929256399460$

$-\odot .48255797717885$

2. 90761224717981

$-1.43221369125712$

4. 39712679107898

$-0.57754054546447$

6. 63845147782556

7.60780176171812

5.44665911823716

6. 37445242024191

$-1.63478594320331$

$-2.57285893436793$

4. 17303615640522
2.16732060941327

2. 16783812641664

2. 16274258231284

2. 16308067608026

2. 15857948707345

2. 15798988080267

2. 15895415384697

2. 15765887080881

2. 15372291817915

2. 15329087873496

2. 14835390219031

2. 14383926032771

2. 14425439617045

2. 14912398119889

2. 14801255987255

2. 14944157914945

2. $1725580955374 \odot$

2. 17206447306537

2. 17649318971700

2. 18141285988499

2. 18197268931506

2. 17754422054773

2.17610879494018

2. 17793084437843

2. 16704275581771

2.16820991611179

2. 13881726165769

2. 13478832378079

2. 16674544650654

2. 16648785874450

2. 16835042128829

2. 16848817138497

2. 14068098425981 
C $\quad-9.86337561217490$

$\mathrm{N} \quad-11.01015048517592$

H $\quad-8.33904802431621$ $\odot .81723974444677$

0.83968347271639

$-1.36650386978133$
2. 18601822826675

2. 18974331200275

2. 18586194043201

\section{Compound $4 / \mathrm{T}_{1}$}

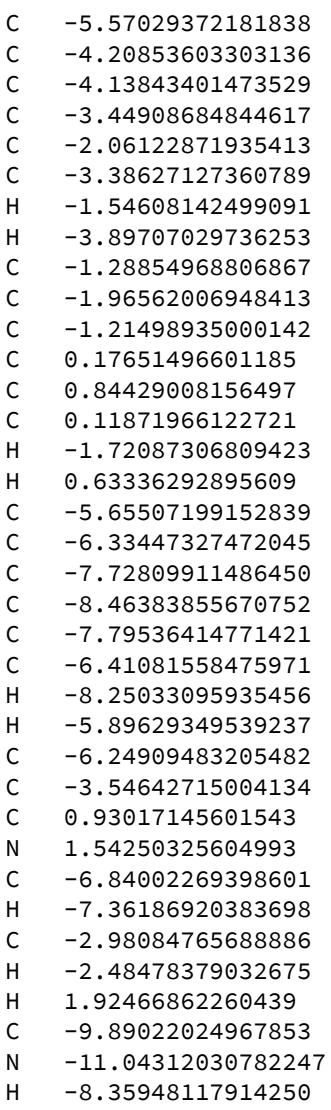

\section{Compound 5 / $\mathrm{S}_{0}$}

3.17309435809928 $\odot .65565111129051$ 3. 14482996619392 1.87002254249260 1. 85912508318740 4. 30963379379668 $\odot .90837424924955$ 5. 26269230666274 3. 04685649476109 4. 30150291519950

5.48438289957266 5.44052366051256

4. 19966248587059

3. 02840885800449 6.44034681095262 2. 07631221187102

$\odot .68707046591741$ 1.93817187107836 1.95057004090079 ค. 75932410687671 $-\odot .46726094961767$ $-0.49244657945878$ 2. 89571414301804 $-1.44228204144208$ 4. 40205058983049 $-\odot .58475402995717$ 6.65296319122837 7.63025283298951

5.45546591296085 6.38052423757536 $-1.65153336188184$ $-2.59057896436615$ 4. 17848228732356 0.80267505478440 $\odot .83550526743033$ $-1.38885262125960$
2. 16724352201804

2. 16795632778342

2. 16262511069042

2. 16298857163810

2. 15847844747626

2. 15777888236751

2. 15874818977611

2. 15749515101348

2. 15351882122051

2. 15315533679708

2. 14827784353747

2. 14382419522302

2. 14418835478422

2. 14896149140741

2. 14796921565654

2. 14923473183914

2. 17264066868207

2. 17229413286601

2. 17684185091568

2. 18170843503095

2. 18204032031027

2. 17754860939051

2. 17661133393333

2. 17781665570174

2. 16692920904046

2. 16832324078688

2. 13888171596202

2. 13489846212662

2.16656362305820

2. 16617687819392

2. 16845172626494

2. 16854102095922

2. 14070401501294

2.18630108411860

2.19001690761683

2. 18578956701819 $-4.21429851006199$ $-4.14768317060092$ $-3.46040259603846$ $-2.05655872807183$ $-3.38896168371148$ $-1.54290633900359$ $-3.90472414409948$ $-1.31651610003685$ $-2.00244471533922$ $-1.23009894752410$ $\odot .13742783089509$ $\odot .82648606948893$ ๑. 10660679018478 $-1.73898769867372$ 0.62759539388834 $-5.62096374578572$ $-6.30709292317473$ $-7.73392193681957$ $-8.43815514131853$ $-7.76181498921880$ $-6.40109781837015$ $-8.24693496721876$ $-9.51977664065697$ $-8.33499597524556$ $-5.88545068068250$ $-6.24499404127706$
3.15940334869060 $\odot .65406891381978$ 3.13078408223552 1. 86148441238452 1.85123817692834 4. 31198313554485 $\odot .900 \odot 9812242331$ 5. 26198215427163 3. 02392583474802 4. 29075125182696 5.48608379221960

5.45320152717169

4. 18063601197931

3. 01744398634592

6.44029715068737

2. 06978078644719

$\odot .69299975110934$

1.96016502039445

1.95983432967291

$\odot .79457392721665$

$-0.45451308367721$

$-\odot .50166883459315$

2. 91042702513788

$\odot .81606876236984$

$-1.37202522761071$

$-1.45083557945804$

4. 40952944373203
2. 16733718336496 2. 16799504641978

2. 16270701183425

2. 16304108306159

2. 15843666055423

2. 15779003176591

2. 15869062133862

2. 15754562175067

2. 15359835703975

2. 15326553767463

2. 14827573431415

2. 14383623238029

2. 14416747825747

2. 14892172706271

2. 14800135052904

2. 14914406751756

2. 17256636351549

2. 17223148153654

2. 17694850702952

2. 18167349224643

2. 18200389507012

2. 17759972842635

2. 17669134931563

2. 18520665355172

2. 18578540608692

2.17784453826184

2. 16699175524269 


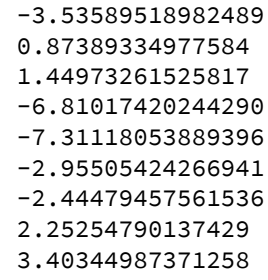

\section{Compound $5 / S_{1}$}

$-\odot .59375246256540$ 6.67544724006747 7.67396410203118 5.47199783938040 6.40837610995090 $-1.64773995693035$ $-2.57910795800358$ 4. 12938374671036

4. 06570874501481
2. 16830314187851 2. 13891443427655 2. 13499456217394 2. 16655105378754 2. 16611735329815 2. 16834398982026 2. 16837634187431 2. 13956971149365

2. 13590408050549
3.15316590152626 ๑. 67042885813484 3.13160259081316 1.86550731135022

1. 85782443332428 4. 30134922041655 $\odot .90853680875949$

5. 25201630338190 3. 03586208770190 4. 29203781467925

5.47313664087235 5.44067157197908

4.19732803069081

3. 02310713845829 6.42769704370301 2. 07757509203039

$\odot .69555740221623$ 1. 94787197180640 1.95529987744765 ๑. 77972105612040 $-0.44370081907238$ $-0.48226526819472$ 2. 90499742817055 $\odot .81152623469514$ $-1.36560224819321$ $-1.43140973231304$ 4. 39401082761778 $-0.57513336320592$ 6.66709566978398

7.66716394307718

5.44853374134252 6. 38251120527938 $-1.62971510745809$ $-2.56491254287005$ 4. 13551432524014 4. 05708417836264
2.16747324142791

2.16791726976164 2. 16286499098868 2. 16319882566909 2. 15869905980056 2. 15810089319627 2. 15910306624701 2. 15777452311589 2. 15380602377844 2. $1533471940674 \odot$

2. 14827857667266 2. 14364220194201

2. 14416333162537

2. 14919393482086

2. 14788658451681 2. 14954379119954

2. 17259479514250 2. 17216209972865 2.17660323548322 2. 18136765176634 2. 18183635510317 2. 17745705520944 2. 17625565080361 2. 18477649117902 $2.1856106980862 \odot$ 2. 17778882998145 2. 16725786127285 2. 16824510851249 2. 13842258152827

2. 13428494558601

2. 16697920041753

2. 16673038970364

2. 16833767264225

2.16843050418078

2. 13947203810026

2. 13576491099946

\section{Compound $5 / \mathrm{T}_{1}$}

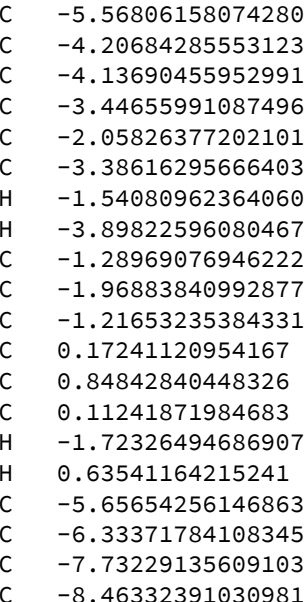

3. 16648996841202 $\odot .65253496753670$ 3. 14028149842443 1.86533125940875 1. 85570144120562 4. 30812572015876 $\odot .90650190241220$ 5. 26024509861998 3. 04655221395465 4. 30085383769773

5. 48417438572921 5.45656493655317

4. 20808107802914

3. 02983858292666 6.43939022148156 2. 08342529892964 $\odot .68443236886048$ 1. 93505528938953 1.94536938132214 $\odot .76265420240314$
2.16730861374597 2. 16796637163764 2. 16264959808713 2. 16298459655665 2.15843206248922 2. 15778764932010 2. 15867186755116 2. 15752890783522 2. 15346447899110 2. 15313429358481

2.14823598899384 2. 14371816558673

2. 14404535442473

2. 14888090591103

2. 14795078904535 2. 14909516504531

2. 17270154270690 2. 17237306427204 2.17698154837844 2. 18183299418332 


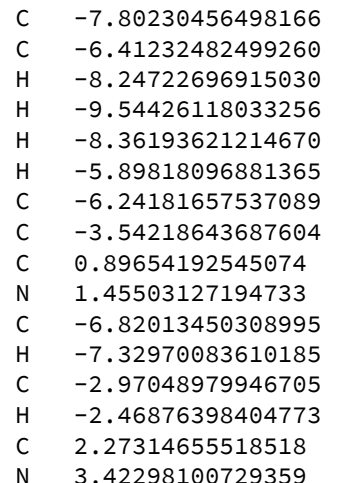

$-\odot .45813856761712$

$-\odot .49239519741796$

2. 89542726602342

$\odot .79988245960864$

$-1.38367733040771$

$-1.44288203215764$

4.39994008297433

$-\odot .58584426288926$

6.68525407227478

7.69382535954689

5.46007160572877

6.39184572105679

$-1.64956036905691$

$-2.58557978978423$

4. 14297263425880

4. 05925032207731
2. 18215382670895

2. 17762184407199

2. 17673522024767

2. 18533601009514

2. 18590972840328

2. 17787385687845

2. 16699108692864

2. 16829530077797

2. 13882350185752

2. 13493947450896

2. 16659365114597

2. 16618256648269

2. 16838093725918

2. 16843896196270

2. 13948462409549

2.13586703448600

\section{Compound 6 / $\mathrm{S}_{0}$}

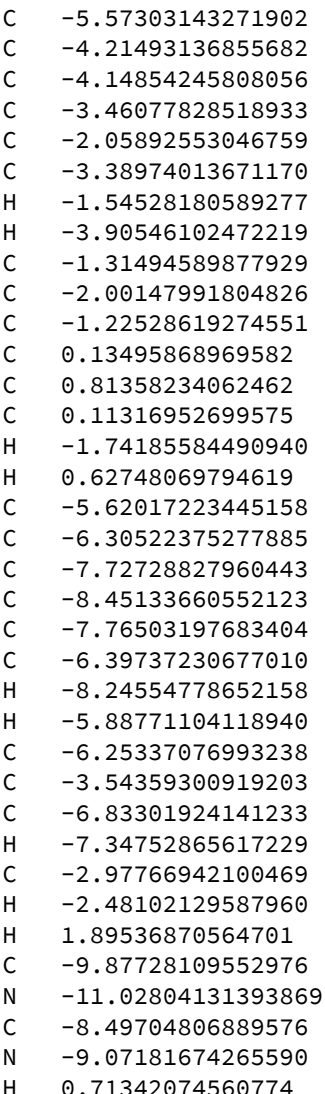

\section{Compound $6 / \mathrm{S}_{1}$}

3. 16031569777644 $\odot .65143016911323$ 3. 13079849409888 1. 86026413042099 1.85110068101451 4. 30956902662349 ๑. 89979259630481 5.25975295561032 3. 02329116871699

4. 29158895856514 5.49036295204830 5.43874255832330

4. 18514058582749

3. 01793359331761

6.44170916980201

2. 06535916890538

$\odot .69460270326257$

1. 96012330456140

1.96051793382201

$\odot .79989166701644$

$-0.46794561935692$

$-0.49629315644545$

2.90764677960940

$-1.44807711309707$

4.40639072215620

$-0.59952215509180$

5.46078246622989

6.38999565819133

$-1.66134332633822$

$-2.60022385902103$

4.16955418462985

$\odot .85679540707175$

$\odot .92182226783910$

$-1.69297869514237$

$-2.69203580832421$

6. 35300984112153
2. 16730071707211

2. 16796376060673

2. 16271843821639

2. 16305612374428

2. 15850732508537

2. 15785295645939

2. 15876450960686

2. 15760513108748

2.15370375779549

2. 15336518928449

2. 14840021832047

2. 14406940261211

2. 14440396275723

2. 14906025959983

2.14815180529115

2. 14931956264785

2.17246515005346

2. 17212951615817

2. 17677056089906

2. 18149333603161

2. 18182787381322

2. 17742055720581

2. 17653808380595

2. 17768908632142

2. 16698059349409

2. 16830034425485

2. 16659302002658

2. 16619587065900

2. 16839460573204

2. 16846478034406

2. 14090848479964

2. 18603322200816

2. 18965728445102

2. 18670198739875

2.19060442938324

2. 14032555165184
C $\quad-5.56217967527823$

C $\quad-4.21153031000534$

C -4.14620492048983

$-3.46310712132599$

$-2.07149188413086$

$-3.39011756700247$

$-1.55825811141652$

$-3.90625790811257$

$-1.30955763808710$

$-1.98755911278682$

$-1.23303031086697$

$\odot .14319889666881$

$\odot .80855858808882$

$\odot .09387578274331$
3. 15639043284045

$\odot .66231930939943$

3. 12591018889152

1. 86479678325699

1.85948616823948

4. 29417741654208

$\odot .90797526540750$

5. 24408775420098

3. 03689795979491

4. 28876402304138

5.47218751501385

5.42751380363379

4. 19884116566352

3. 02192996483914
2. 16736234020084

2. 16781799932762

2. 16282165226407

2. 16312757806253

2. 15866391524850

2. 15805236586501

2. 15903391149880

2. 15773531937043

2. 15382941696793

2. 15338004738033

2. 14837453286078

2. 14388595424730

2. 14434954853319

2. 14925347202480 


$\begin{array}{ll}\mathrm{H} & -1.74837997720814 \\ \mathrm{H} & 0.60840169389618 \\ \mathrm{C} & -5.6312198334750 \odot \\ \mathrm{C} & -6.31185747273994 \\ \mathrm{C} & -7.71277529696652 \\ \mathrm{C} & -8.44536781414213 \\ \mathrm{C} & -7.77509009655297 \\ \mathrm{C} & -6.39218883085390 \\ \mathrm{H} & -8.23319850038017 \\ \mathrm{H} & -5.88614622905854 \\ \mathrm{C} & -6.23995918332500 \\ \mathrm{C} & -3.54372254260979 \\ \mathrm{C} & -6.82307848423172 \\ \mathrm{H} & -7.34438908178410 \\ \mathrm{C} & -2.98590106156829 \\ \mathrm{H} & -2.49732688448465 \\ \mathrm{H} & 1.88889681221241 \\ \mathrm{C} & -9.86969283527121 \\ \mathrm{~N} & -11.01342609571590 \\ \mathrm{C} & -8.49805350801540 \\ \mathrm{~N} & -9.05700696319301 \\ \mathrm{H} & \odot .71279068887962\end{array}$

\section{Compound $6 / \mathrm{T}_{1}$}

6.42304765608096 2. 07070018806202

$\odot .69357080133924$

1.95064626943385

1. 95703180955814

$\odot .78396892287966$

$-0.45438937933946$

$-\odot .48273613431301$

2.90178395846936

$-1.43527262364912$

4. 39653480829630

$-\odot .58310300105547$

5.44457414854695

6. 37001458225536

$-1.64484166285058$

$-2.58797191842286$

4. 17405504807243

$\odot .85135473715969$

$\odot .93196558807267$

$-1.68342619335685$

$-2.68453154811174$

6.34561130127072
2.14801561144016

2. 14960836158482

2. 17248962654855

2. 17206091086408

2. 17650540017061

2. 18133516009097

2. 18183950741776

2. 17737050575589

2. 17618719731892

2. 17771440256544

2. 16710033655231

2. 16813325131536

2. 16682109282636

2. 16657691402649

2. 16825429678109

2. 16837991828898

2. 14080365030385

2. 18584222900238

2. 18940835038679

2. 18682792819271

2. 19079902568845

2. 13997572770512
3. 16956579948285

$\odot .65035719508202$

3. 13942764248246

1. 86580778590481

1. 85803961936253

4. 30535567517138

๑. 90767256862214

5. 25734915070763

3. 04841142180989

4. 30146316307629

5.48737563578275

5.44414937653937

4. 21285421273610

3. 03182539916589

6.43953596586170

2. 07974199737664

0.68574473837355

1.93860197479206

1.94945348513915

$\odot .76945556300484$

$-\odot .46843517612523$

$-0.48903443820697$

2. 89490768874974

$-1.44233865603329$

4. 39841725557781

$-\odot .59238131328401$

5.44829095947131

6.37021814883140

$-1.66581596096206$

$-2.61139182627000$

4.18607085316187

$\odot .84462738189825$

๑. 93603144801248

$-1.70173113667391$

$-2.71397795860434$

6.36421946914620
2. 16725373630414

2. 16791699990873

2. 16265275905295

2. 16298992369607

2. 15848884672141

2. 15783971146673

2. 15873613905572

2. 15758363081820

2. 15355935436625

2. 15322572639163

2. 14836249887865

2. 14394765323056

2. 14427574360681

2. 14901652790696

2. 14810897592717

2. 14927008901189

2. 17257836469874

2. 17224772494180

2. 17678916385266

2. 18162578836667

2. 18195193780646

2. 17743181308336

2. 17657208873682

2. 17771436899991

2. 16697227989708

2. 16828585489929

2. 16665552910113

2. 16632023026042

2. 16845614004476

2. 16859387676328

2. 14080812501512

2. 18612744400211

2. 18969754008477

2. 18679641255230

2. 19065675823227

2. 14022770099640

\section{Compound 7 / $\mathrm{S}_{0}$}

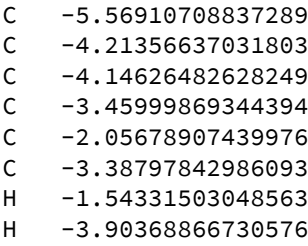

3.16350413505525 $\odot .65375428240638$ 3. 13243515806368 1. 86251746717341 1.85154343146333 4. 31379822415306 $\odot .90039445342324$ 5. 26376555230331
2. 16726535242934

2. 16796430095570

2. 16266451926917

2. 16301776604548

2. 15844004176511

2. 15775797128615

2. 15870562202398

2. 15750245141034 


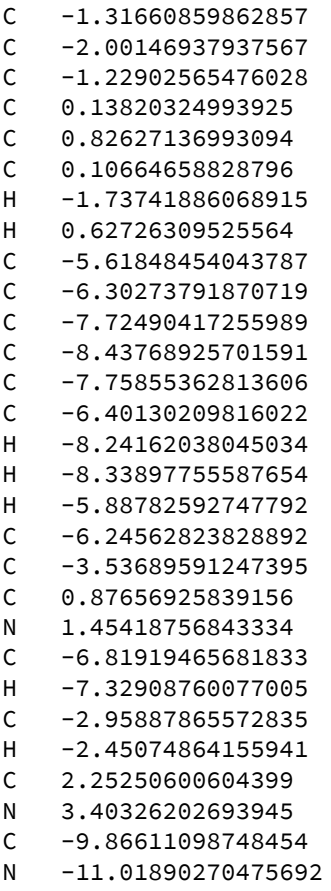

3. 02454147286782

4. 29162624664465

5.48716177248018

5. 45283393609239

4.17993079731069

3. 01682006569891

6.44155418889228

2. 06904685429693

0.69461841409364

1. 96153617287803

1. 96804304133431

0.79840417402776

$-\odot .45961795849292$

$-\odot .49951857565803$

2. 91593412195626

$-1.37106664361962$

$-1.44965777049338$

4.41120807263845

$-0.59469318341168$

6.67410200742967

7.67140854738503

5.46888461371905

6.40088649721629

$-1.64995944013977$

$-2.58277767799190$

4. 12883946971025

4. 06534572843986

$\odot .82552744154395$

$\odot .83132859383258$
2.15360963006978

2. 15325811842125

2. 14827692408801

2. 14386380200575

2. 14421372861695

2. 14895842119654

2. 14798964499460

2. 14919337649697

2. 17250216155395

2. 17214719221475

2. 17681063132836

2. 18156250590909

2. 18191643297281

2. 17753256865105

2. 17656027421335

2. 18570128386013

2. 17779743294834

2. 16691883016241

2. 16829397130581

2. 13894654871416

2. 13503015715089

2. 16650290589345

2. 16609922365225

2. 16835847647131

2. 16841271576540

2. 13963849641919

2. 13599194639655

2.18620652880141

2. 18998758217360

\section{Compound $7 / S_{1}$}

\begin{tabular}{|c|c|}
\hline & -5.55399562985536 \\
\hline & -4.21027995438737 \\
\hline & -4.14256897130113 \\
\hline & -3.45649965757047 \\
\hline & -2.06701888071552 \\
\hline & -3.38802923968405 \\
\hline & -1.55211370343633 \\
\hline & $-3.9 \odot 111506509383$ \\
\hline & -1.30778444261847 \\
\hline & -1.98727448565676 \\
\hline & -1.23182239028157 \\
\hline & $\odot .14846101841865$ \\
\hline & $\odot .82149169889989$ \\
\hline & $\odot . \odot 9297455761868$ \\
\hline & -1.73707568922374 \\
\hline & $\odot .61492583643112$ \\
\hline & -5.63034793843877 \\
\hline & -6.31040877752848 \\
\hline & $-7.707270907 \odot 8768$ \\
\hline & -8.43265690927720 \\
\hline & -7.76557735410404 \\
\hline & $-6.39178193010 \odot 95$ \\
\hline & -8.22655834704366 \\
\hline & -8.33584925675885 \\
\hline & -5.87947772981051 \\
\hline & -6.23367259727379 \\
\hline & -3.54218287450334 \\
\hline & $\odot .87787676348693$ \\
\hline & 1.44100524552362 \\
\hline & -6.81500419533440 \\
\hline & -7.33343312071871 \\
\hline & -2.97392799803242 \\
\hline & -2.47230481945278 \\
\hline & 2.24626004323272 \\
\hline & 3. 38995192453885 \\
\hline & -9.8591613883917 \\
\hline & \\
\hline
\end{tabular}

3.15795529759740 $\odot .67020530731592$

3. 13316513525280

1. 86704253022911

1.85805972338268

4. $30236616860 \odot 88$

0.90821320738764

5. 25320909829372

3. 03693257485454

4. 29197498774367

5.47328679101913

5.43928309924319

4. 19539052171044

3. 02167908811902

6.42822157424111

2. 07582085237603

0.69699706739713

1. 95082339392602

1.96366651443830

$\odot .78208696404150$

$-\odot .44762902323391$

$-\odot .48178324553453$

2. 90953645501909

$-1.36442274454762$

$-1.43168647237997$

4. 39648877544904

$-\odot .57559025979900$

6.66524942733498

7.66356086159195

5.44503248132413

6.37278403474175

$-1.63121130995444$

$-2.56798990880666$

4.13378748832028

4. 05652921181637

0.82061123421618

$\odot .84435678199545$
2.16742326937017

2.16789054173312

2. 16283961816289

2. 16316877425064

2. 15869575455218

2. 15807539949731

2. 15909985441753

2. 15774503106515

2. 15380379485587

2. 15334259089199

2. 14828091050300

2. 14366975109697

2. 14419456480887

2. 14921406530090

2. 14788113919519

2. 14956778994854

2. 17257347216595

2. 17210948667455

2.17646646240447

2. 18129810996627

2. 18185196066094

2. 17749033230017

2. 17608646178149

2. 18568212279208

2. 17786996329851

2. 16721765104870

2. 16820544336523

2. 13846254912697

2.13433582004613

2. 16698697948456

2. 16679338751705

2.16828765139490

2. 16837345167548

2. 13952180202873

2. 13582819495321

2. 18580939265017

2. 18945599264749

\section{Compound $7 / \mathrm{T}_{1}$}


๑. 65450982483026

3.14391311715636

1.86910830704139

1.85731368511409

4. 31143619105906

$\odot .90761988624305$

5. 26385770774206

3. 04799211846571

4. 30228013842879

5.48529472295127

5.45525115974003

4. 20591928407704

3. 02861031888488

6.44115051101798

2. 08160573167833

$\odot .68690943698054$

1.93799440571746

1.95243879747005

$\odot .76193936241026$

$-\odot .46519375805650$

$-\odot .49213413537965$

2.89793368010182

$-1.38627196440875$

$-1.44255780748679$

4. 40216400595781

$-0.58482189011129$

6.68285891640719

7.68985606418477

5.45569611581015

6.38116767495706

$-1.64973629664591$

$-2.58729370553140$

4.13983978377111

4.05550920300998

$\odot .80711573301640$

$\odot .84234038775258$
2. 16793271850945

2. 16260431010739

2. 16295559461343

2. 15843497976300

2. 15775019285973

2. 15868941658704

2.15747828861243

2. 15347272250553

2. 15312438246403

2. 14823240528268

2. 14374415550732

2. 14409056946372

2. 14891865052463

2. 14793084731924

2. 14914882017806

2. 17262882382338

2. 17228990120380

2. 17684786822772

2. 18172147935952

2. 18204714873791

2. 17754383194821

2. 17662217887143

2. 18580207770622

2. 17780891758401

2. 16692115578588

2. 16828392604871

2. 13885222379584

2. 13496968657072

2. 16656410102208

2. 16619798278468

2. 16839371534350

2. 16847573317595

2. 13955490692927

2. 13595792794013

2. 18632622051900

2. 19004991414440

\section{Compound 8 / $\mathrm{S}_{0}$}

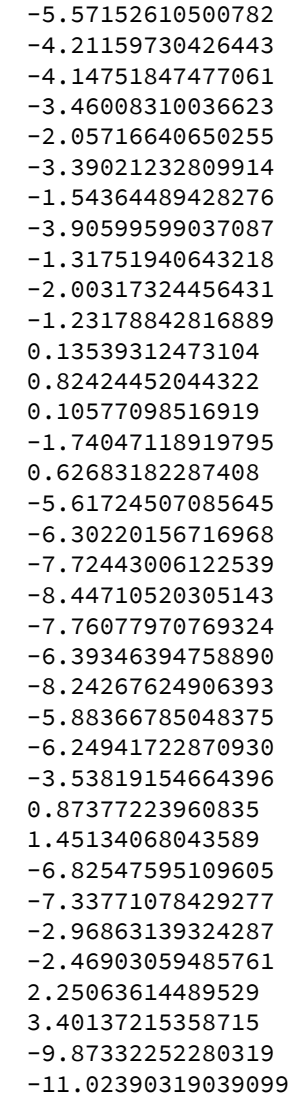

3. 16312464016118 $\odot .65151002399033$ 3. 13109309364537 1. 86148712947496 1.85014052342260 4. 31210797454339 ๑. 89905083438008 5.26197345961129

3. 02371037689337

4. 29004565725702

5.48620468252040

5.45185592405567

4.17965872621067

3. 01596199722459

6.44036597684400

2. 06850129827953

$\odot .69644297813651$

1.96146742934777

1.96283465561522

$\odot .80173050300642$

$-\odot .46582273328207$

$-\odot .49528804989725$

2. 90987721555887

$-1.44690658981897$

4.40974428614063

$-\odot .59754040846153$

6.67324753443652

7.67044033246950

5.46585617189494

6.39683972454275

$-1.65717096451126$

$-2.59499479591490$

4. 13045189221964

4. 06890493960775

$\odot .85663151456527$

$\odot .91999651090772$
2. 16725881862724

2. 16792260302241

2. 16266073112063

2.16299743000055

2. 15842672208630

2. 15777415330558

2.15868322663982

2. 15752792916820

2. 15361257441464

2. 15327678728746

2. 14831344146829

2. 14390468256104

2. 14423918067661

2. 14896619459570

2. 14803620806307

2. 14919055391001

2. 17244824998226

2. 17211254902045

2. 17678037605207

2. 18152736379099

2. 18186207553565

2. 17743125918284

2. 17654743338047

2.17769978227153

2. 16693147967514

2. 16825254432104

2. 13900270794893

2. 13509943679374

2. 16653642677608

2. 16615155890288

2. 16834239682004

2. 16842527708836

2. 13966396183445

2. 13601678731145

2. 18610065915190

2. 18975105713639 
C -8.49435814852933

N $\quad-9.07020692158252$
$-1.69014660035694$ $-2.68826769188505$
2. 18676873888942

2. 19069521427338

Compound $8 / S_{1}$

\begin{tabular}{|c|c|}
\hline & -5.55670588059218 \\
\hline & -4.20810728179901 \\
\hline & -4.14285315438464 \\
\hline & -3.45715787040841 \\
\hline & -2.06763646138568 \\
\hline & -3.39083610205906 \\
\hline & -1.55272271394218 \\
\hline & -3.90495188740117 \\
\hline & -1.30950243497645 \\
\hline & -1.98855396151653 \\
\hline & -1.23574726926707 \\
\hline & $\odot .14449999387640$ \\
\hline & $\odot .81805571402357$ \\
\hline & $\odot .09139843812478$ \\
\hline & -1.74172987299260 \\
\hline & $\odot .61400253714058$ \\
\hline & -5.62886683865405 \\
\hline & -6.30848914565026 \\
\hline & -7.70744453965148 \\
\hline & -8.43962160169277 \\
\hline & -7.76922150773159 \\
\hline & -6.38607079816562 \\
\hline & -8.22830616481018 \\
\hline & -5.87996521182837 \\
\hline & -6.23714586600600 \\
\hline & -3.54304332336488 \\
\hline & $\odot .87399648852 \odot 91$ \\
\hline & 1.43775195767311 \\
\hline & -6.82023601595977 \\
\hline & -7.34094920189314 \\
\hline & -2.98526445952553 \\
\hline & -2.49483806325390 \\
\hline & $2.2433786132810 \odot$ \\
\hline & 3.38695957210496 \\
\hline & -9.86419766056895 \\
\hline & -11.00762896470604 \\
\hline & -8.49267921898554 \\
\hline & -9.05272298113714 \\
\hline
\end{tabular}

3. 15679356428788

$\odot .66751067433559$

3. 13126595702469

1. 86564882990309

1.85705412477497

4. 29971426994616

$\odot .90704242736224$

5.25011013788280

3.03649542400192

4. 29026004446838

5.47181530105637

5.43863761442962

4. 19473717636550

3. 02091973351274

6. 42640562263506

2.07539925582730

$\odot .69647656853270$

1.95111578251576

1.96012609833576

๑.78649744796662

$-\odot .45146553016095$

$-\odot .47990905838140$

2.90476817919197

$-1.43255092154124$

4. 39487252581951

$-\odot .57883896872357$

6. 66457051088918

7.66231744080915

5.44226073729421

6.36894705761725

$-1.63994129198448$

$-2.58299933666131$

4. 13508898320905

4.06076337491836

$\odot .85278346847262$

$\odot .93130296951096$

$-1.68044597962124$

$-2.68043004298721$
2.16736588660791

2. 16777472186829

2. 16279377910274

2. 16307580754204

2. 15860199717044

2. 15806961985844

2. 15896623208430

2. 15777531846041

2. 15377210437731

2. 15335585433916

2. 14835399464044

2. 14377617263841

2. 14426043841709

2. 14920621833278

2. 14798290168111

2. 14952258636334

2. 17251178787973

2. 17201273997879

2. 17632682400032

2. 18121059773151

2. 18192040311950

2. 17751002131229

2.17587753906038

2. 17799221660633

2. 16720587506640

2. 16802618174369

2. 13863853188213

2. 13456773993727

2. 16701787137832

2. 16686563564717

2. 16808226301672

2. 16815032214253

2. 13961396002555

2. 13593879721275

2. 18557401117263

2. 18902326494881

2. 18706130843163

2.19115704730832

\section{Compound $8 / \mathrm{T}_{1}$}

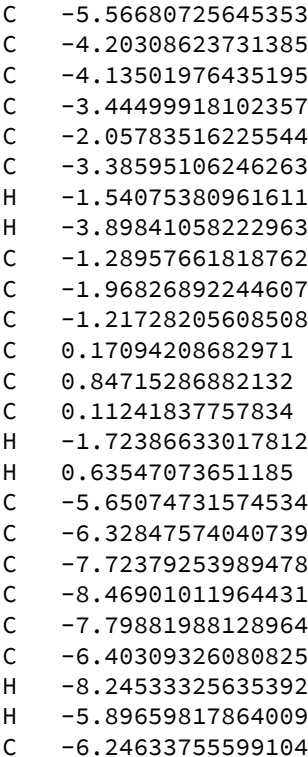

3. 17020826044476 ๑. 65160552246695

3. 14095378454868

1. 86658819535434

1. 85569215373288

4.30853352892650

$\odot .90624004921537$

5. 26048868966908

3. 04718583630267

4. 30063828362121

5.48467175452024

5.45598510604742

4. 20713094691069

3. 02892907792855

6.43989714289143

2. 08261951371563

$\odot .68694409524955$

1.93860840991787

1. 95091898651091

$\odot .77059920076897$

$-0.46714597996739$

$-0.48823463904326$

2. 89617354039561

$-1.44163622524498$

4. 39921677854483
2. 16722364931217

2. 16788621368049

2. 16259620384694

2. 16293291223968

2. 15841383243903

2. 15776539494961

2. 15865655958170

2. 15750636925833

2. 15347172650484

2. 15313968189726

2. 14826924786659

2. 14378276164848

2. 14411229416897

2. 14891887221789

2. 14798200236283

2. 14913451382510

2.17257459493046

2. 17224441057833

2. 17680528588861

2. 18166560133524

2.18199160997328

2. 17744799675893

2. 17658928367931

2. 17773149091613

2. 16693405169314 
$\quad-3.54531735804704$ $\odot .89642639194426$

1.45642133754703

$-6.83875748840669$

$-7.36259603426190$

$-2.98819169929577$

$-2.50033993143285$

2. 27217376352446

3.42184396180374

$-9.89303842218691$

$-11.04208445442952$

$-8.51480408203288$

$-9.06680836265369$
$-0.58918577767912$

6.68415989724757

7.69154930053202

5.45116619950217

6.37568462725373

$-1.66024643721351$

$-2.60424967814713$

4.14338824949398

4. 06160555864772

$\odot .84507480037648$

$\odot .93589446109249$

$-1.70034056842690$

$-2.71219247327201$
2. 16824632216620

2. 13890944074370

2. 13504211006129

2. 16660433797600

2. 16627370971947

2. 16840537193472

2. 16854629731934

2. 13957490616312

2. 13597549453564

2. 18619416222261

2.18978529250386

2. 18686250712113

2. 19074205906658 
[1] S. N. Sanders, E. Kumarasamy, A. B. Pun, M. L. Steigerwald, M. Y. Sfeir, L. M. Campos, Angew. Chemie Int. Ed. 2016, 55, 3373.

[2] C. Sánchez-Sánchez, A. Nicolaï, F. Rossel, J. Cai, J. Liu, X. Feng, K. Müllen, P. Ruffieux, R. Fasel, V. Meunier, J. Am. Chem. Soc. 2017, 139, 17617.

[3] A. B. Pun, S. N. Sanders, E. Kumarasamy, M. Y. Sfeir, D. N. Congreve, L. M. Campos, Adv. Mater. 2017, 29, 1701416.

[4] S. H. C. Askes, W. Pomp, S. L. Hopkins, A. Kros, S. Wu, T. Schmidt, S. Bonnet, Small 2016, 12, 5579.

[5] J. C. de Mello, H. F. Wittmann, R. H. Friend, Adv. Mater. 1997, 9, 230.

[6] E. Kumarasamy, S. N. Sanders, M. J. Y. Tayebjee, A. Asadpoordarvish, T. J. H. Hele, E. G. Fuemmeler, A. B. Pun, L. M. Yablon, J. Z. Low, D. W. Paley, J. C. Dean, B. Choi, G. D. Scholes, M. L. Steigerwald, N. Ananth, D. R. McCamey, M. Y. Sfeir, L. M. Campos, J. Am. Chem. Soc. 2017, 139, 12488. 